H. Omoto and S. Nakano

Nagoya Math. J

Vol. 75 (1979), 41-70

\title{
LOCAL DEFORMATIONS OF ISOLATED SINGULARITIES ASSOCIATED WITH NEGATIVE LINE BUNDLES OVER ABELIAN VARIETIES
}

\author{
HIDEO OMOTO and SHIGEO NAKANO
}

\section{Introduction}

Let $V$ be an analytic space with an isolated singularity $p$. In [1] M. Kuranishi approached the problem of deformations of isolated singularities (c.f. [2] and [3]) as follows; Let $M$ be a real hypersurface in the complex manifold $V-\{p\}$. Then one has the induced $C R$-structure ${ }^{\circ} T^{\prime \prime}(M)$ on $M$ by the inclusion map $i: M \rightarrow V-\{p\}$ (c.f. Def. 1.6). Then deformations of the isolated singularity $(V, p)$ give rise to ones of the induced $C R$-structure ${ }^{\circ} T^{\prime \prime}(M)$. He established in $\S 9$ in [1] the universality theorem for deformations of the induced $C R$-structure ${ }^{\circ} T^{\prime \prime}(M)$, when $M$ is compact strongly pseudo-convex (Def. 1.5) of $\operatorname{dim} M \geqq 5$. Form this theorem we can know $C R$-structures on $M$ which appear in deformations of ${ }^{\circ} T^{\prime \prime}(M)$.

Here we assume that $V$ is 1-convex in the sense of Andoreotti-Grauert such that $\operatorname{dim}_{C} V \geqq 3$ and that $M$ is a compact real hypersurface in $V$ $\{p\}$ defined by strictly plurisubharmonic function $\rho$ on $V$ such that $\rho \geqq 0$, that is, $M=\{q \in V ; \rho(q)=c\}$, here $c$ is a constant. Then as $\operatorname{Prof}_{p} V \geqq 2$, we find in terms of [2] that the infinitesimal deformation $H^{1}(V, \Theta)$ (c.f. [1]) of the isolated singularity $(V, p)$ is regarded as a subspace of the infinitesimal deformation $H^{1}\left(M,{ }^{\circ} T^{\prime \prime}(M)\right.$ ) of ${ }^{\circ} T^{\prime \prime}(M)$ (c.f. $\left.\S 3\right)$. Therefore in order to solve the problem of local deformations of $(V, p)$, it is enough to determine the infinitesimal deformations $H^{1}\left(M,{ }^{\circ} T^{\prime \prime}(M)\right)$ and complex structure on a neighborhood of $M$ in $V-\{p\}$, which induce $C R$-structures on $M$ appearing in deformations of ${ }^{\circ} T^{\prime \prime}(M)$.

In this paper we shall prove, using the above Kuranishi's theory, the following.

Received February 28, 1978.

The first named author was a visiting member at the Research Institute for Mathematical Sciences, Kyoto University, during a part of the period of this research. 
TheOREm 5.1. Let $T$ be an abelian variety of $\operatorname{dim} T \geqq 2$ and $B$ a negative line bundle over $T . \quad$ Let $(B, T)$ be the isolated singularity defined by the exceptional variety $O(T)$ in $B$, here $O(T)$ denotes the zero section of $B$. Then local deformations of $(B, T)$ are also isolated singularities $\left(B^{\prime}, T^{\prime}\right)$, where $T^{\prime}$ and $B^{\prime}$ are abelian varieties and negative line bundles over $T^{\prime}$, respectively.

Remark. $(B, T)$ is 1-convex with $\operatorname{dim}_{C} B \geqq 3$.

The above theorem has been essentially proved by M. Schlessinger [2]. However we want to publish this paper as an example of applications of Kuranishi's theory to deformations of isolated singularities.

In $\S 1$ we describe basic notions of $C R$-structures and in $\S 2$ determine the induced $C R$-structure ${ }^{\circ} T^{\prime \prime}\left(B_{1}\right)$ on the unit sphere bundle $B_{1}$ of the negative line bundle $B$ over the abelian variety $T$ using a normalized automorphic factor for $B$. In $\S 3$ we calculate the infinitesimal deformation $H^{1}\left(B,{ }^{\circ} T^{\prime \prime}(B)\right)$.

In $\S 4$ and $\S 5$ we show that $H^{1}\left(B,{ }^{\circ} T^{\prime \prime}(B)\right)$ has basis which are integrable $C R$-structures and that these integrable $C R$-structures are induced from some negative line bundles $B^{\prime}$ over abelian varieties $T^{\prime}$.

\section{$\S 1$ Basic definitions}

Let $M$ be a real oriented smooth manifold of dimension $2 n+1, n=$ $1,2, \cdots$, and let ${ }^{\circ} T^{\prime \prime}$ be a subbundle of the complexified tangent bundle $C T M$. Let $E$ be a vector bundle over $M$. We denote by $\Gamma(E)$ the set of $C^{\infty}$-sections of $E$.

Definition 1.1. The subbundle ${ }^{\circ} T^{\prime \prime}$ is called an almost $C R$-structure on $M$, when the following condition is satisfied;

$$
{ }^{\circ} T^{\prime \prime} \cap{ }^{\circ} \bar{T}^{\prime \prime}=0, \quad \operatorname{dim}_{c}^{\circ} T^{\prime \prime}=n .
$$

Moreover an almost $C R$-structure ${ }^{\circ} T^{\prime \prime}$ is a $C R$-structure, provided that

${ }^{\circ} T^{\prime \prime}$ is integrable in the sense of Frobenius, i.e. if $Z_{1}, Z_{2}$ are sections of ${ }^{\circ} T^{\prime \prime}$, then so is their Lie bracket $\left[Z_{1}, Z_{2}\right]$.

Now let ${ }^{\circ} T$ ' be a $C R$-structure on $M$. Since ${ }^{\circ} T$ ' is the complex vector subbundle of $C T M$ of complex fiber dimension $n$ and is invariant under complex conjugation, there is a real line bundle $F$ of $T M$ such that 


$$
C T M={ }^{\circ} T^{\prime \prime} \oplus{ }^{\circ} \bar{T}^{\prime \prime} \oplus C F .
$$

From now on we fix this decomposition of $C T M$. Put

$$
T^{\prime}={ }^{\circ} T^{\prime \prime} \oplus C F,
$$

and we denote by $\pi^{\prime}(\pi)$ the projection of $C T M$ onto $T^{\prime}\left({ }^{\circ} T^{\prime \prime}\right)$, respectively.

Definition 1.2. An almost $C R$-structure $E^{\prime \prime}$ on $M$ is said to be of finite distance to ${ }^{\circ} T^{\prime \prime}$ if $\pi^{\prime} \circ i_{E^{\prime \prime}}: E^{\prime \prime} \rightarrow T^{\prime}$ is an isomorphism, where $i_{E^{\prime \prime}}: E^{\prime \prime}$ $\rightarrow C T M$ is the inclusion map.

Proposition 1.3 [1]. Let $E^{\prime \prime}$ be an almost $C R$-structure on $M$ of finite distance to ${ }^{\circ} T^{\prime \prime}$. Then there is a unique element $\varphi$ of $\Gamma\left(M, \operatorname{Hom}\left({ }^{\circ} T^{\prime \prime}, T^{\prime}\right)\right)$ such that

$$
E^{\prime \prime}=\left\{X-\varphi(X) ; X \in^{\circ} T^{\prime \prime}\right\} .
$$

Conversely let $\varphi \in \Gamma\left(M, \operatorname{Hom}\left({ }^{\circ} T^{\prime \prime}, T^{\prime}\right)\right)$ and we write

$$
\varphi=\varphi_{1}+\varphi_{2}
$$

where $\varphi_{1} \in \Gamma\left(M, \operatorname{Hom}\left({ }^{\circ} T^{\prime \prime},{ }^{\circ} \bar{T}^{\prime \prime}\right)\right)$ and $\varphi_{2} \in \Gamma\left(M, \operatorname{Hom}\left({ }^{\circ} T^{\prime \prime}, C F\right)\right)$. For any $\varphi$ satisfying $\varphi_{1} \overline{\left(\varphi_{1}(X)\right)} \neq \bar{X}$ or $\varphi_{2} \overline{\left(\varphi_{1}(X)\right)} \neq \overline{\varphi_{2}(X)}$ for all $X \in{ }^{\circ} T^{\prime \prime},(X \neq 0)$, the formula (1.5) defines an almost CR-structure $E^{\prime \prime}$ of finite distance to ${ }^{\circ} T^{\prime \prime}$.

The almost $C R$-structure $E^{\prime \prime}$ defined by (1.5) in terms of $\varphi$ will be denoted by ${ }^{\varphi} T^{\prime \prime}$. We identify ${ }^{\varphi} T^{\prime \prime}$ with $\varphi$, and $\varphi$ is also called an (almost) $C R$-structure on $M$, when ${ }^{\prime} T$ " is an (almost) $C R$-structure.

Next we will examine when $T^{\prime \prime}$ is integrable, i.e., a $C R$-structure on $M$. For any $X \in \Gamma(C T M)$ we put

$$
X_{T^{\prime}}=\pi^{\prime}(X) \quad \text { and } \quad X_{{ }^{\prime \prime} T^{\prime \prime}}=\pi(X) .
$$

The following formulation for the integrable condition is due to T. Akahori [4].

Proposition 1.4. Let $\varphi \in \Gamma\left(\mathrm{Hom}\left({ }^{\circ} T^{\prime \prime}, T^{\prime}\right)\right)$ be an almost $C R$-structure on $M$. Let $P(\varphi)$ be a map of $\Gamma\left(\bigwedge^{2}{ }^{\circ} T^{\prime \prime}\right)$ into $\Gamma\left(T^{\prime}\right)$ defined by

$$
\begin{array}{r}
P(\varphi)(X, Y)=[X-\varphi(X), Y-\varphi(Y)]_{T^{\prime}}+\varphi\left([X-\varphi(X), Y-\varphi(Y)]_{\circ T^{\prime \prime}}\right) \\
\text { for } X, Y \in \Gamma\left(^{\circ} T^{\prime \prime}\right) .
\end{array}
$$

Then $\varphi$ is integrable if and only if

$$
P(\varphi) \equiv 0 \text {. }
$$


In order to compute the infinitesimal deformation of ${ }^{\circ} T^{\prime \prime}$, we must define the operator $\bar{\partial}_{b}^{(p)}: \Gamma\left(T^{\prime} \otimes \bigwedge^{p}\left({ }^{\circ} T^{\prime \prime}\right)^{*}\right) \rightarrow \Gamma\left(T^{\prime} \otimes \bigwedge^{p+1}\left({ }^{\circ} T^{\prime \prime}\right)^{*}\right), p=0,1,2, \cdots$. At first for $p=0, \bar{\partial}_{b}^{(0)}: \Gamma\left(T^{\prime}\right) \rightarrow \Gamma\left(T^{\prime} \otimes{ }^{\circ} T^{\prime \prime}\right)$ is defined by

$$
\left(\bar{\partial}_{b}^{(0)} u\right)(X)=[X, u]_{T^{\prime}}, \quad \text { for } u \in \Gamma\left(T^{\prime}\right) \text { and } X \in \Gamma\left({ }^{\circ} T^{\prime \prime}\right) \text {. }
$$

For convenience sake we set

$$
X u=[X, u]_{T^{\prime}}
$$

For $p \geqq 1$,

$$
\begin{aligned}
\left(\bar{\partial}_{b}^{(p)} \psi\right) & \left(X_{1}, \cdots, X_{p+1}\right) \\
= & \sum_{i=1}^{p}(-1)^{i-1} X_{i} \psi\left(X_{1}, \cdots, \hat{X}_{i}, \cdots, X_{p+1}\right) \\
& \quad+\sum_{i<j}(-1)^{j+k} \psi\left(\left[X_{i}, X_{j}\right], X_{1}, \cdots, \hat{X}_{i}, \cdots, \hat{X}_{j}, \cdots, X_{p+1}\right),
\end{aligned}
$$

where $X_{1}, \cdots, X_{p+1} \in \Gamma\left({ }^{\circ} T^{\prime \prime}\right)$, and $\psi \in \Gamma\left(T^{\prime} \otimes \Lambda^{p}\left({ }^{\circ} T^{\prime \prime}\right)^{*}\right)$. From definitions of $\bar{\partial}_{b}^{(p)}$ it is clear that $\bar{\partial}_{b}^{(p+1)} \cdot \bar{\partial}_{b}^{(p)}=0$, i.e.,

$$
0 \longrightarrow \Gamma\left(T^{\prime}\right) \stackrel{\bar{\partial}_{b}^{(0)}}{\longrightarrow} \Gamma\left(T^{\prime} \otimes\left({ }^{\circ} T^{\prime \prime}\right)^{*}\right) \stackrel{\bar{\partial}_{b}^{(1)}}{\longrightarrow}\left(T^{\prime} \otimes \wedge^{2}\left({ }^{\circ} T^{\prime \prime}\right)^{*}\right) \longrightarrow \cdots
$$

is a complex.

We explain the convexity of the $C R$-structure ${ }^{\circ} T^{\prime \prime}$ on $M$. Let $X_{1}, \cdots$, $X_{n}$ be a frame of $\Gamma\left(^{\circ} \bar{T}^{\prime \prime} \mid U\right)$ for some open set $U$ in $M$. Then $\bar{X}_{1}, \cdots, \bar{X}_{n}$ become the frame of $\Gamma\left({ }^{\circ} T^{\prime \prime} \mid U\right)$. Take a cross-section $S$ of $\Gamma(F \mid U)$ such that $S(p) \neq 0$ for any $p \in U$. Hence we obtain smooth functions $C_{\jmath k}$ on $U$ defined by

$$
\begin{aligned}
\sqrt{-1}\left[X_{j}, \bar{X}_{k}\right] \equiv C_{j k} S\left(\bmod { }^{\circ} T^{\prime \prime} \oplus{ }^{\circ} \bar{T}^{\prime \prime}\right), \\
\\
\quad \text { for } 1 \leq j, k \leq n .
\end{aligned}
$$

It is trivial that the functional matrix

$$
\left\|C_{j k}\right\| \text { is hermitian . }
$$

Definition 1.5. ${ }^{\circ} T^{\prime \prime}$ is strongly pseudo-convex, when for any $p \in M$, there is a cross-section $S$ of $\Gamma(F \mid U)$ such that

$$
\left\|C_{j k}\right\|>0, \quad \text { on some neighborhood } U \text { of } P \text {. }
$$

Finally let us consider a complex manifold $V$ and an imbedding $i: M$ $\rightarrow V$, where $\operatorname{dim}_{C} V=n+1$ and $\operatorname{dim}_{R} M=2 n+1$. Then a complex subbundle ${ }^{\circ} T^{\prime \prime}$ on $M$ is defined as follows: For any point $p \in M$, 


$$
{ }^{\circ} T_{p}^{\prime \prime}=C i_{p^{*}} T M \cap T_{i(p)} V,
$$

where $T_{i(p)} V$ denotes the holomorphic tangent space of $V$ at $i(P)$. Since $i(M)$ is a real hypersurface of $V,{ }^{\circ} T^{\prime \prime}(M)$ becomes the subbundle of $C T M$ of complex fiber dimension $n$. Clearly ${ }^{\circ} T^{\prime \prime}$ is integrable, i.e., this subbundle defines a $C R$-structure on $M$.

Definition 1.6. Let $i: M \rightarrow V$ be an imbedding as above. Then the $C R$-structure ${ }^{\circ} T^{\prime \prime}$ on $M$ defined by (1.10) is called the induced $C R$ structure by $i$, or simply the induced $C R$-structure.

\section{§2. CR-structures on negative line bundles over abelian varieties}

2.1. Let $T$ be an abelian variety with an $n \times 2 n$-matrix $\omega=$ $\left(\omega_{\alpha}^{i}\right)_{1 \leq i \leq n, 1 \leq \alpha \leq 2 n}$ as a period matrix, that is, let $C^{n}$ be the space of $n$ complex variables $\left(z_{1}, \cdots, z_{n}\right)$ and let $Z^{2 n}=\overbrace{Z \times \cdots \times Z}^{2 n}$. The elements of $C^{n}$ and $Z^{2 n}$ are written as column vectors of length $n$ and $2 n$, respectively. For any element $\boldsymbol{d}={ }^{t}\left(d^{1}, \cdots, d^{2 n}\right)$ of $Z^{2 n}$, we put

$$
\boldsymbol{\omega} \cdot \boldsymbol{d}={ }^{t}\left(\sum_{\alpha=1}^{2 n} \omega_{\alpha}^{1} d^{\alpha}, \cdots, \sum_{\alpha=1}^{2 n} \omega_{\alpha}^{n} d^{\alpha}\right) .
$$

if $\Lambda$ denotes the lattice $\left\{\omega \cdot \boldsymbol{d} ; \boldsymbol{d} \in Z^{2 n}\right\}$ in $\boldsymbol{C}^{n}$, then

$$
T=C^{n} / \Lambda \text {. }
$$

Now let $B$ be a negative line bundle over $T$ and let $\pi$ be the projection of $C^{n}$ onto $T$. Then the induced bundle $\pi^{-1}(B)$ of $B$ under $\pi$ is isomorphic to the trivial bundle $C^{n} \times C$. From this fact there exists a holomorphic map $f: C^{2 n} \times Z^{2 n} \rightarrow C-\{0\}$, called an "automorphic factor" of $B$, satisfying the following conditions (6);

$$
\begin{aligned}
& \text { For } d_{1}, d_{2} \text { in } Z^{2 n} \text {, and } z \in C^{n}, \\
& f\left(z, d_{1}+d_{2}\right)=f\left(z+\omega d_{1}, d_{2}\right) f\left(z, d_{2}\right) .
\end{aligned}
$$

(C.2) let $\sim$ be the equivalence relation in $C^{n} \times C$ defined by

$$
\begin{aligned}
& \left(z_{1}, \zeta_{1}\right) \sim\left(z_{2}, \zeta_{2}\right) \Longleftrightarrow \text { there is } d \in Z^{2 n} \text { such that } \\
& \left(z_{2}, \zeta_{2}\right)=\left(z_{1}+\omega d, f\left(z_{1}, d\right) \zeta_{1}\right) .
\end{aligned}
$$

Then the line bundle $B$ over $T$ is isomorphic to $C^{n} \times C / \sim$. It is clear that automorphic factors of $B$ depend on the choice of isomorphisms of $\pi^{-1}(B)$ onto $C^{n} \times C$. And we can take an automorphic factor $f$ of $B$ which has the following form (c.f. pp. 111, [5]); 
For any $z \in C^{n}$ and $d \in Z^{2 n}$,

$$
f(z, d)=\exp \left\{2 \pi \sqrt{-1}\left({ }^{t} z Q \bar{\omega} d+\frac{1}{2} d A^{\circ} d+{ }^{t} b \cdot d\right)\right\}
$$

where

$$
Q=\left[\begin{array}{ccc}
Q_{11}, & \cdots, & Q_{1 n} \\
\vdots & & \vdots \\
Q_{n 1}, & \cdots, & Q_{n n}
\end{array}\right]
$$

is an $(n \times n)$-matrix such that ${ }^{t} \bar{Q}=-Q$, and $A^{\circ}$ is a $(2 n \times 2 n)$-matrix and $b$ denotes a real column vector of length $2 n$. We fix the above automorphic factor $f$ with (2.1). Let $h: C^{n}: \rightarrow R$ be the smooth positive function defined by

$$
h(z)=\exp \left(-2 \pi \sqrt{-1}{ }^{t} z Q \bar{z}\right) .
$$

Then we have $h(z)=h(z+\omega d)|f(z, d)|^{2}$ for every $d \in Z^{2 n}$ and $z \in C^{n}$, so that $h$ induces the hermitian metric $\tilde{h}$ on the line bundle $B$ over $T$. Hence the Chern class $c(B)$ of $B$ equals to the de Rham cohomology class of

$$
\left(\frac{1}{2 \pi \sqrt{-1}} \bar{\partial} \log h=\sum_{i, j=1}^{n} Q_{i \bar{j}} d z^{j} \wedge d \bar{z}^{j}\right) .
$$

However since $B$ is negative, we have $\sqrt{-1} Q<0$, that is, the hermitian matrix $\sqrt{-1} Q$ is negative definite.

2.2. From the negativity of $B$ we know that if $T$ is regarded as the zero-section of $B$, then there exists an analytic variety $\tilde{T}$ and a holomorphic map $g$ of $B$ onto $\tilde{T}$ such that for some point $\tilde{t}_{0} \in \tilde{T}$.

$g$ is a bi-holomorphic map of $B-T$ onto $\tilde{T}-\left\{\tilde{t}_{0}\right\}$, and $g(T)=\tilde{t}_{0}$. (c.f. [6])

Clearly $\tilde{T}$ has the isolated singularity point $\tilde{t}_{0}$, which is denoted by $\left(\tilde{T}, \tilde{t}_{0}\right)$. Let $\tilde{S}$ be a real hypersurface around $\tilde{t}_{0}$ in $\tilde{T}-\left\{\tilde{t}_{0}\right\}$. Then local deformations of isolated singularity $\left(\tilde{T}, \tilde{t}_{0}\right)$ induce ones of the induced $C R$ structure on $\tilde{S}$ by the inclusion $i_{\tilde{S}}: \tilde{S} \rightarrow \tilde{T}-\left\{\tilde{t}_{0}\right\}$. However in terms of the biholomorphic map $\mathrm{g}: B-T \rightarrow \tilde{T}-\left\{\tilde{t}_{0}\right\}$ we shall consider local deformations of the induced $C R$-structure on a real hypersurface around $T$ in $B-T$.

Now let $B_{1}$ be the unit circle bundle over $T$ defined by the hermitian metric $h$ on $B$, i.e.,

$$
B_{1}=\{e \in B ; \tilde{h}(e)=1\}
$$


Proposition 2.1. Let ${ }^{\circ} T^{\prime \prime}\left(B_{1}\right)$ be the induced CR-structure on $B_{1}$ by the inclusion $\iota_{B_{1}}: B_{1} \rightarrow B$. Then ${ }^{\circ} T^{\prime \prime}\left(B_{1}\right)$ is strongly pseudo-convex, that is, $B_{1}$ is the real $(2 n+1)$-dimensional compact strongly pseudo-convex manifold.

This proposition is proved by the following two lemmas. At first let $\tilde{\psi}$ be the natural projection of $C^{n} \times C$ onto $B=C^{n} \times C / \sim$ as in $\S 1$. Here put

$$
V=\tilde{\psi}^{-1}\left(B_{1}\right)\left(\subset C^{n} \times C\right)
$$

Then it is trivial that

$$
V=\left\{(z, \zeta) \in C^{n} \times C ; h(z)|\zeta|^{2}=1\right\} .
$$

Moreover let $\psi$ be the diffeomorphism of $C^{n} \times S^{1}$ onto $V$ defined by

$$
\psi(z, \theta)=\left(z, \frac{e^{\sqrt{-1 \theta}}}{\sqrt{h(z)}}\right), \quad \text { for }(z, \theta) \in C^{n} \times S^{1},
$$

here $\theta$ is the angular coordinate of $S^{1}$.

Lemma 2.2. Let ${ }^{\circ} T^{\prime \prime}(V)=C T V \cap T^{0,1}\left(C^{n} \times C\right)$ be the induced $C R$ structure on $V$. Let $Z_{\overline{1}}, \cdots$, and $Z_{n}$, be vector fields on $C^{n} \times S^{1}$ defined by

$$
Z_{\bar{j}}=\frac{\partial}{\partial \bar{z}^{j}}-\frac{\sqrt{-1}}{2} \frac{\partial \log h}{\partial \bar{z}^{j}} \frac{\partial}{\partial \theta}, \quad j=1, \cdots, n .
$$

Then $\left\{\psi_{*}\left(Z_{\bar{j}}\right)\right\}_{j=1}^{n}$ become the global basis of ${ }^{\circ} T^{\prime \prime}(V)$.

Proof. By direct calculations we obtain

$$
\psi_{*(z, \theta)}\left(\frac{\partial}{\partial \bar{z}^{j}}\right)=\frac{\partial}{\partial \bar{z}^{j}}-\frac{1}{2} \frac{\partial \log h}{\partial \bar{z}^{j}} \frac{e^{\sqrt{-1} \theta}}{\sqrt{h}} \frac{\partial}{\partial \zeta}-\frac{1}{2} \frac{\partial \log h}{\partial \bar{z}^{j}} \frac{e^{-\sqrt{-1} \theta}}{\sqrt{h}} \frac{\partial}{\partial \bar{\zeta}}
$$

and

$$
\psi_{*(z, \theta)}\left(\frac{\partial}{\partial \theta}\right)=\sqrt{-1}\left(\frac{e^{\sqrt{-1} \theta}}{\sqrt{h}} \frac{\partial}{\partial \zeta}-\frac{e^{-\sqrt{-1} \theta}}{\sqrt{h}} \frac{\partial}{\partial \bar{\zeta}}\right)
$$

Here we have

$$
\psi_{*}\left(Z_{\bar{j}}\right)=\frac{\partial}{\partial \bar{z}^{j}}-\bar{\zeta} \frac{\partial \log h}{\partial \bar{z}^{j}} \frac{\partial}{\partial \bar{\zeta}}, \quad j=1, \cdots, n
$$

This means $\psi_{*(z, \theta)}\left(Z_{\bar{j}}\right) \in T_{\psi(z, \theta)}^{0,1}\left(C^{n} \times C\right)$, for any $(z, \theta) \in C^{n} \times S^{1}$, so that the $\psi_{*}\left(Z_{j}\right)$ are cross-sections of ${ }^{\circ} T^{\prime \prime}(V)$. It is clear that $\left\{\psi^{*}\left(Z_{j}\right)\right\}_{j=1}^{n}$ is the global base of ${ }^{\circ} T^{\prime \prime}(V)$.

Q.E.D. 
Next let ${ }^{\circ} T^{\prime \prime}\left(C^{n} \times S^{1}\right)$ be the complex subspace of $C T\left(C^{n} \times S^{1}\right)$ generated by the basis $\left\{Z_{j}\right\}_{j=1}^{n}$. Then from the above lemma it is trivial that ${ }^{\circ} T^{\prime \prime}\left(C^{n} \times S^{1}\right)={ }^{\circ} T^{\prime \prime}(V)$, and that ${ }^{\circ} T^{\prime \prime}\left(C^{n} \times S^{1}\right)$ is the $C R$-structure on $C^{n} \times S^{1} . C T\left(C^{n} \times S^{1}\right)$ has the following decomposition; let $F=\{R(\partial / \partial \theta)\}$ be the real line bundle over $C^{n} \times S^{1}$, spanned by $\partial / \partial \theta$. Then we get

$$
C T\left(C^{n} \times S^{1}\right)={ }^{\circ} T^{\prime \prime}\left(C^{n} \times S^{1}\right) \oplus{ }^{\circ} \bar{T}^{\prime \prime}\left(C^{n} \times S^{1}\right) \oplus C F \text {. }
$$

LEMMA 2.3. ${ }^{\circ} T^{\prime \prime}\left(C^{n} \times S^{1}\right)$ is strongly convex.

Proof. Put $Z_{j}=\bar{Z}_{\bar{j}}(j=1, \cdots, n)$. By (2.3) and (2.2) it follows that

$$
\left[Z_{\bar{j}}, Z_{j}\right]=\sqrt{-1} \frac{\partial^{2} \log h}{\partial \bar{z}^{j} \partial z^{j}} \frac{\partial}{\partial \theta}=2 \pi Q_{i \bar{j}} \frac{\partial}{\partial \theta} \quad(1 \leq i, j \leq n)
$$

On the other hand since $Q$ is negative definite, ${ }^{\circ} T^{\prime \prime}\left(C^{n} \times S^{1}\right)$ is strongly convex.

Q.E.D.

Remark. The next formulas are trivial;

$$
\begin{aligned}
{\left[Z_{\bar{j}}, Z_{\bar{i}}\right]=\left[Z_{j}, Z_{i}\right]=\left[Z_{\bar{j}}, \frac{\partial}{\partial \theta}\right]=\left[Z_{j}, \frac{\partial}{\partial \theta}\right] } & =0 \\
& \text { for } 1 \leq i, j \leq n .
\end{aligned}
$$

We shall express the induced $C R$-structure ${ }^{\circ} T^{\prime \prime}\left(B_{1}\right)$ by using $\left\{Z_{\bar{j}}\right\}_{j=1}^{n}$. Let $\tilde{\psi}$ be the canonical projection of $C^{n} \times C$ onto $B=C^{n} \times C / \sim$ and $\hat{\psi}$ the composite map of $\psi$ and $\tilde{\psi}$;

$$
\hat{\psi}=\tilde{\psi} \circ \psi: C^{n} \times S^{1} \stackrel{\psi}{\longrightarrow} C^{n} \times C \stackrel{\psi}{\longrightarrow} B .
$$

It follows from (C.2) in 2.1 and the definition of $\psi$ that for $(z, \theta),\left(z^{\prime}, \theta^{\prime}\right) \in$ $C^{n} \times S^{1}, \hat{\psi}(z, \theta)=\hat{\psi}\left(z^{\prime}, \theta^{\prime}\right)$ means that there is a $d \in Z^{2 n}$ such that

$$
z^{\prime}=z+\omega d, \text { and } \theta^{\prime}=\theta+\arg f(z, d) .
$$

LemMa 2.4. For $(z, \theta) \in C^{n} \times S^{1}$, and $d \in Z^{2 n}$

$$
\hat{\psi}_{*(z, \theta)}\left(Z_{\bar{j}}\right)=\hat{\psi}_{*(z+\omega d, \theta+\arg f(z, d))}\left(Z_{\bar{j}}\right), \quad(j=1, \cdots, n),
$$

and

$$
\hat{\psi}_{*(z, \theta)}\left(\frac{\theta}{\partial \theta}\right)=\hat{\psi}_{*(z+\omega d, \theta+\arg f(z, d))}\left(\frac{\partial}{\partial \theta}\right)
$$

Therefore $\left\{\hat{\psi}_{*}\left(Z_{\bar{j}}\right)\right\}_{j=1, \ldots, n}$ and $\hat{\psi}_{*}(\partial / \partial \theta)$ become vector fields on $B_{1}$.

Proof. It follows that 


$$
\begin{aligned}
& \hat{\psi}_{*(z+\omega d, \theta+\arg f(z, d))}\left(Z_{j}\right)
\end{aligned}
$$

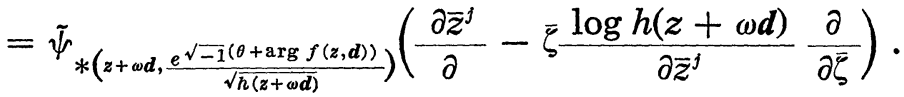

Here set

$$
\zeta_{0}=\frac{e^{\sqrt{-1}(\theta+\arg f(z, d))}}{\sqrt{h(z+\omega d)}}
$$

and

$$
A=\hat{\psi}_{*((z+\omega d, \theta+\arg f(z, d)))}\left(Z_{\mathrm{j}}\right) .
$$

Using $h(z)=h(z+\omega d)|f(z, d)|^{2}$ as in $\S 1$, we get

$$
A=\tilde{\psi}_{*\left(z+\omega d, \zeta_{0}\right)}\left(\frac{\partial}{\partial \bar{z}^{j}}+\frac{\partial \log f(z, d)}{\partial \bar{z}^{j}} \bar{\zeta} \frac{\partial}{\partial \bar{\zeta}}-\frac{\partial \log h(z)}{\partial \bar{z}^{j}} \bar{\zeta} \frac{\partial}{\partial \bar{\zeta}}\right) .
$$

On the other hand let $g_{d}$ be the bi-holomorphic map of $C^{n} \times C$ onto $C^{n}$ $\times C$ defined by

$$
g_{d}(z, \zeta)=(z+\omega d, f(z, d) \zeta), \quad \text { for any } d \in Z^{2 n} .
$$

Then we have

$$
g_{d\left(z, f(z, d)-1 \zeta_{0}\right)}\left(\frac{\partial}{\partial \bar{z}^{j}}\right)=\left(\frac{\partial}{\partial \bar{z}^{j}}+\frac{\partial \log f(z, d)}{\partial \bar{z}^{j}} \zeta_{0}\left(\frac{\partial}{\partial \zeta}\right)\right)_{g d\left(z, f(z, d)-1 \zeta_{0}\right.},
$$

and

$$
g_{d\left(z, f(z, d)-1 \zeta_{0}\right)}\left(\bar{\zeta} \frac{\partial}{\partial \bar{\zeta}}\right)=\bar{\zeta}_{0}\left(\frac{\partial}{\partial \bar{\zeta}}\right)_{g d\left(z, f(z, d)-1 \zeta_{0}\right)} .
$$

Hence from (2.7) it follows that

$$
\left.A=\left(\tilde{\psi} \cdot g_{d}\right)_{\left(z, f(z, d)-1 \xi_{0}\right)}\left(\psi_{*(z, \theta)} Z_{\bar{j}}\right) \quad \text { (c.f. }(2.4)\right)
$$

But as $\tilde{\psi} g_{d}=\tilde{\psi}$, we have finally

$$
A=\hat{\psi}_{*(z, \theta)} Z_{\bar{j}}
$$

Similarly it is proved

$$
\hat{\psi}_{*(z, \theta)}\left(\frac{\partial}{\partial \theta}\right)=\hat{\psi}_{*(z+\omega d, \theta+\arg f(z, d))}\left(\frac{\partial}{\partial \theta}\right) .
$$

Q.E.D.

Let us return to the proof of Proposition 2.1. By virtue of Lemmas 2.2 and 2.4. the induced $C R$-structure ${ }^{\circ} T^{\prime \prime}\left(B_{1}\right)$ on $B_{1}$ is spanned by $\hat{\psi}_{*}\left(Z_{l}\right)$, 
$\cdots$, and $\hat{\psi}_{*}\left(Z_{\bar{n}}\right)$, denoted by $\left\{\left\{\hat{\psi}_{*}\left(Z_{\overline{1}}\right), \cdots, \hat{\psi}_{*}\left(Z_{\bar{n}}\right)\right\}\right.$. Furthermore we have the next decomposition of $C T B_{1}$;

$$
C T B_{1}={ }^{\circ} T^{\prime \prime}\left(B_{1}\right) \oplus{ }^{\circ} \bar{T}^{\prime \prime}\left(B_{1}\right) \oplus C F\left(B_{1}\right),
$$

where

$$
\left\{\begin{array}{l}
{ }^{\circ} T^{\prime \prime}\left(B_{1}\right)=\left\{\left\{\hat{\psi}_{*}\left(Z_{\overline{1}}\right), \cdots, \hat{\psi}_{*}\left(Z_{\bar{n}}\right),\right\}\right\}, \\
{ }^{\circ} \bar{T}^{\prime \prime}\left(B_{1}\right)=\left\{\left\{\hat{\psi}_{*}\left(Z_{1}\right), \cdots, \hat{\psi}_{*}\left(Z_{n}\right)\right\}\right\}, \\
C F\left(B_{1}\right)=\left\{\left\{\hat{\psi}_{*}(\partial / \partial \theta)\right\}\right\} .
\end{array}\right.
$$

Thus our proposition is completely proved.

\section{§3. Infinitesimal deformations of ${ }^{\circ} T^{\prime \prime}\left(B_{1}\right)$}

Notations being as in $\S 2$, let us first consider relations between almost $C R$-structures of finite distance to ${ }^{\circ} T^{\prime \prime}\left(C^{n} \times S^{1}\right)$ on $C^{n} \times S^{1}$ (c.f. Lemma 2.3) and ones of finite distance to ${ }^{\circ} T^{\prime \prime}\left(B_{1}\right)$ on $B_{1}$. For this purpose we set

$$
{ }^{\circ} T^{\prime}\left(C^{n} \times S^{1}\right)={ }^{\circ} \bar{T}^{\prime \prime}\left(C^{n} \times S^{1}\right) \oplus C F
$$

and

$$
T^{\prime}\left(B_{1}\right)={ }^{\circ} \bar{T}^{\prime \prime}\left(B_{1}\right) \oplus C F\left(B_{1}\right) .
$$

From Proposition 1.3 it is enough to consider the correspondence between $\Gamma\left(\operatorname{Hom}\left({ }^{\circ} T^{\prime \prime}\left(C^{n} \times S^{1}\right), T^{\prime}\left(C^{n} \times S^{1}\right)\right)\right)$ and $\Gamma\left(\operatorname{Hom}\left({ }^{\circ} T^{\prime \prime}\left(B_{1}\right), T^{\prime}\left(B_{1}\right)\right)\right)$. Here we put for simplicity

$$
{ }^{\circ} T^{\prime \prime}={ }^{\circ} T^{\prime \prime}\left(C^{n} \times S^{1}\right) \text { and } T^{\prime}=T^{\prime}\left(C^{n} \times S^{1}\right) .
$$

Let $Z_{\overline{1}}, \cdots$, and $Z_{n}$ be the basis of ${ }^{\circ} T^{\prime \prime}$ defined by Lemma 2.2. Then we have the following

Proposition 3.1. For any $\varphi \in \Gamma\left(\operatorname{Hom}\left({ }^{\circ} T^{\prime \prime}, T^{\prime}\right)\right)$ we can write

$$
\varphi\left(Z_{\mathfrak{j}}\right)=\sum_{k=1}^{n} \varphi_{\bar{j}}^{k} Z_{k}+\varphi_{j} \frac{\partial}{\partial \theta} \quad(j=1, \cdots, n),
$$

where $\varphi_{\bar{j}}^{k}$ and $\varphi_{j}$ are smooth functions on $C^{n} \times S^{1}$. Then $\varphi$ induces an element of $\Gamma\left(\operatorname{Hom}\left({ }^{\circ} T^{\prime \prime}\left(B_{1}\right), T^{\prime}\left(B_{1}\right)\right)\right)$ if and only if the following condition (C) is satisfied;

$$
\text { (C) }\left\{\begin{array}{l}
\varphi_{\bar{j}}^{k}(z, \theta)=\varphi_{\bar{j}}^{k}(z+\omega d, \theta+\arg f(z, d)) \\
\varphi_{j}(z, \theta)=\varphi_{j}(z+\omega d,+\arg f(z, d))
\end{array}\right.
$$


for each $d \in Z^{2 n}$ and $(z, \theta) \in C^{n} \times S^{1}$.

Proof. Let $\tau$ be the linear map from $\Gamma\left(\operatorname{Hom}\left({ }^{\circ} T^{\prime \prime}\left(B_{1}\right), T^{\prime}\left(B_{1}\right)\right)\right)$ to $\Gamma\left(\operatorname{Hom}\left({ }^{\circ} T^{\prime \prime}, T^{\prime}\right)\right)$ defined as follows; let $\tilde{\varphi}$ be any element of $\Gamma\left(\operatorname{Hom}\left({ }^{\circ} T^{\prime \prime}\left(B_{1}\right)\right.\right.$, $\left.T^{\prime}\left(B_{1}\right)\right)$ ). Then we put, for any $(z, \theta) \in C^{n} \times S^{1}$,

$$
\left[(\tau \tilde{\varphi})\left(Z_{\bar{j}}\right)\right]_{(z, \theta)}=\hat{\psi}_{* \hat{\psi}}^{-1}(z, \theta)\left(\tilde{\varphi}\left(\hat{\psi}_{*(z, \theta)}\left(Z_{\bar{j}}\right)\right)\right) .
$$

If $\tilde{\varphi}$ is an element of $\Gamma\left(\operatorname{Hom}\left({ }^{\circ} T^{\prime \prime}\left(B_{1}\right), T^{\prime}\left(B_{1}\right)\right)\right)$ with the expression

$$
\tilde{\varphi}\left(\hat{\psi}_{*}\left(Z_{\bar{j}}\right)=\sum_{k=1}^{n} \tilde{\varphi}_{\bar{j}}^{k} \hat{\psi}_{*}\left(Z_{k}\right)+\tilde{\varphi}_{j} \hat{\psi}_{*}\left(\frac{\partial}{\partial \theta}\right),\right.
$$

then it follows that

$$
(\tau \hat{\varphi})\left(Z_{\bar{j}}\right)=\sum_{k=1}^{n}\left(\tilde{\varphi}_{\bar{j}}^{k} \circ \hat{\psi}\right) Z_{k}+\left(\tilde{\varphi}_{j} \circ \hat{\psi}\right) \frac{\partial}{\partial \theta}
$$

Thus $\tau \tilde{\varphi}$ satisfies the condition (C). Conversely an arbitrary element $\varphi \in \Gamma\left(\operatorname{Hom}\left({ }^{\circ} T^{\prime \prime}, T\right)\right)$ satisfying (C) induces an element $\tilde{\varphi}$ of $\Gamma\left(\operatorname{Hom}\left({ }^{\circ} T^{\prime \prime}\left(B_{1}\right)\right.\right.$, $\left.\left.T^{\prime}\left(B_{1}^{1}\right)\right)\right)$, and we have

$$
\varphi=\tau \tilde{\varphi} .
$$

We denote by $\Gamma_{(C)}\left(\operatorname{Hom}\left({ }^{\circ} T^{\prime \prime}, T^{\prime}\right)\right)$ the set of all smooth-section of Hom $\left({ }^{\circ} T^{\prime \prime}, T^{\prime}\right)$ satisfying the condition (C). The above Proposition 3.1 shows that

$$
\tau: \Gamma\left(\operatorname{Hom}\left({ }^{\circ} T^{\prime \prime}\left(B_{1}\right), T^{\prime}\left(B_{1}\right)\right)\right) \rightarrow \Gamma_{(C)}\left(\operatorname{Hom}\left({ }^{\circ} T^{\prime \prime}, T^{\prime}\right)\right)
$$

is isomorphic.

More generally let $\Gamma_{(C)}\left(\bigwedge^{k}\left({ }^{\circ} T^{\prime \prime}\right)^{*} \otimes T^{\prime}\right)$ be the set of all smoothsections $\varphi$ of $\wedge^{k}\left({ }^{\circ} T^{\prime \prime}\right)^{*} \otimes T^{\prime},(k=0,1, \cdots, n)$ such that, when $\varphi$ is expressed as

$$
\begin{aligned}
&\left(Z_{\bar{j}_{1}} \wedge \cdots \wedge Z_{\bar{j}_{k}}\right)=\sum_{\ell=1}^{n} \varphi_{\bar{j}_{1}, \ldots, \bar{j}_{k}}^{\ell} Z_{\bar{l}}+\varphi_{j_{1}, \ldots, j_{k}} \frac{\partial}{\partial \theta} \\
&\left(1 \leq j_{1}<\cdots<j_{k} \leq n\right) .
\end{aligned}
$$

Then all coefficients $\varphi_{j_{1}, \ldots, \bar{j}_{k}}$ and $\varphi_{j_{1}, \ldots, j_{k}}$ satisfy the condition (C). Then $\tau$ induces the isomorphism of $\Gamma\left(\bigwedge^{k}\left({ }^{\circ} T^{\prime \prime}\left(B_{1}\right)\right)^{*} \otimes T^{\prime}\left(B_{1}\right)\right)$ onto $\Gamma_{(C)}\left(\bigwedge^{k}\left({ }^{\circ} T^{\prime \prime}\right)^{*}\right.$ $\left.\otimes T^{\prime}\right)$. Here we have the following commutative diagram; 


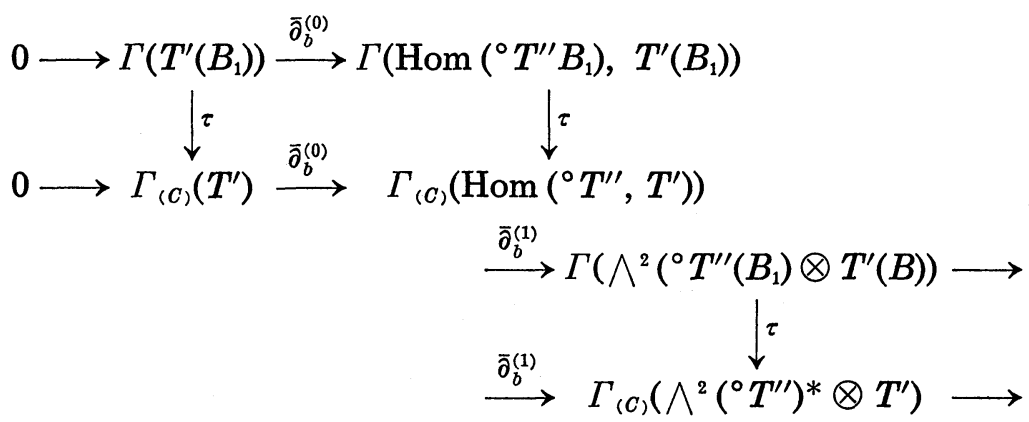

where the $\bar{\partial}_{b}^{(k)}$ denote the operators defined by (1.7) and (1.8).

Let $H^{k}\left({ }^{\circ} T^{\prime \prime}\left(B_{1}\right)\right)$ and $H_{(C)}^{k}\left({ }^{\circ} T^{\prime \prime}\right)$ be the $k$-th cohomologies of complexes $\left\{\Gamma\left(\bigwedge^{k}\left({ }^{\circ} T^{\prime \prime}\left(B_{1}\right)\right)^{*} \otimes T^{\prime}\left(B_{1}\right)\right), \overline{\hat{\sigma}}_{b}^{(k)}\right\}_{k=0}^{n}$ and $\left\{\Gamma_{(C)}\left(\bigwedge^{k}\left({ }^{\circ} T^{\prime \prime}\right)^{*} \otimes T^{\prime}\right), \bar{\partial}_{b}^{(k)}\right\}_{k=0}^{n}$, respectively. Then we know that

$$
H^{k}\left({ }^{\circ} T^{\prime \prime}\left(B_{1}\right)\right) \cong H_{(C)}^{k}\left({ }^{\circ} T^{\prime \prime}\right) .
$$

3.1. We shall determine explicitly a basis of the first cohomology $H_{(C)}^{1}\left({ }^{\circ} T^{\prime \prime}\right)$, that is, the infinitesimal deformation of ${ }^{\circ} T^{\prime \prime}\left(B_{1}\right)$. First of all let $\varphi$ be an element of $\Gamma_{(C)}\left(\operatorname{Hom}\left({ }^{\circ} T^{\prime \prime}, T^{\prime}\right)\right.$ with

$$
\varphi\left(Z_{j}\right)=\sum_{k=1}^{n} \varphi_{\bar{j}}^{k} Z_{k}+\varphi_{j} \frac{\partial}{\partial \theta} \quad(j=1, \cdots, n) .
$$

Then we obtain the following

Proposition 3.2. It follows that $\bar{\partial}_{b}^{(1)} \varphi=0$ if and only if for all $i$, $j \in\{1, \cdots, n\}$,

$$
Z_{\bar{i}} \varphi_{\bar{j}}^{k}-Z_{\bar{j}} \varphi_{i}^{k}=0, \quad(k=1, \cdots, n)
$$

and

$$
\sum_{k=1}^{n}\left(\varphi_{\bar{j}}^{k} \Phi_{k \bar{i}}-\varphi_{\bar{i}}^{k} \Phi_{k \bar{j}}\right)+Z_{\bar{i}} \varphi_{j}-Z_{\bar{j}} \varphi_{i}=0
$$

where we put

$$
\left.\Phi_{i \bar{j}}=2 \pi Q_{i \bar{j}} \quad \text { (c.f. }(2.1)\right) .
$$

Proof. By (1.8) and (2.6) we see that

$$
\begin{aligned}
\bar{\partial}_{b}^{(1)} \varphi\left(Z_{\bar{i}}, Z_{\bar{j}}\right)= & {\left[Z_{\bar{i}}, \varphi\left(Z_{\bar{j}}\right)\right]_{T^{\prime}}-\left[Z_{\bar{j}}, \varphi\left(Z_{\bar{i}}\right)\right]_{T^{\prime}} } \\
= & \sum_{k=1}^{n}\left(Z_{\bar{i}} \varphi_{\bar{j}}^{k}-Z_{\bar{j}} \varphi_{\bar{i}}^{k}\right) Z_{k}+\sum_{k=1}^{n}\left(\varphi_{\bar{j}}^{k}\left[Z_{\bar{i}}, Z_{k}\right]_{T^{\prime}}-\varphi_{\bar{i}}^{k}\left[Z_{\bar{j}}, Z_{k}\right]_{T^{\prime}}\right) \\
& +\left(Z_{\bar{i}} \varphi_{j}-Z_{\bar{j}} \varphi_{i}\right) \frac{\partial}{\partial \theta} .
\end{aligned}
$$


However as $\left[Z_{\bar{j}}, Z_{i}\right]=2 \pi Q_{i j}(\partial / \partial \theta)$ (c.f. $\left.(2.5)\right)$, it is trivial that

$$
\begin{aligned}
\bar{\partial}_{\bar{b}}^{(1)} \varphi\left(Z_{\bar{i}}, Z_{\bar{j}}\right)= & \sum_{n=1}^{n}\left(Z_{\bar{i}} \varphi_{\bar{i}}^{k}-Z_{\bar{j}} \varphi_{\bar{i}}^{k}\right) Z_{k} \\
& +\left\{\sum_{k=1}^{n}\left(\varphi_{\bar{j}}^{k} 2 \pi Q_{k \bar{j}}-\varphi_{\bar{i}}^{k} 2 \pi Q_{k \bar{i}}\right)+Z_{\bar{i}} \varphi_{j}-Z_{\bar{j}} \varphi_{i}\right\} \frac{\partial}{\partial \theta} .
\end{aligned}
$$

This fact proves Proposition 3.2.

Q.E.D.

In order to study properties of the $\left\{\varphi_{j}^{k}, \varphi_{j}\right\} k, j=1, \cdots, n$ on $C^{n} \times S^{1}$ satisfying the differential equations (3.2) and (3.3), we denote by $\subseteq(f)$ the Fourier expansion of any function $f$ on $C^{n} \times S^{1}$ with respect to the angular parameter of $S^{1}$. Let us put

$$
\Im\left(\varphi_{\bar{j}}^{k}\right)(z, \theta)=\sum_{m \in Z} \varphi_{\bar{j}, m}^{k}(z) e^{\sqrt{-1} m \theta} .
$$

Then from the uniqueness of Fourier expansions and the condition (C) it follows that

$$
\varphi_{\bar{j}, m}^{k}(z+\omega d)=\varphi_{\bar{j}, m}^{k}(z) e^{-\sqrt{-1} m \arg f(z, d)}
$$

for any $d \in Z^{2 n}$ and $m=0, \pm 1, \pm 2, \cdots$.

At first we consider the differential systems (3.2). By (2.3), (3.2) means that

$$
\begin{aligned}
\frac{\partial \varphi_{\bar{j}}^{k}}{\partial \bar{z}^{i}}-\frac{\partial \varphi_{i}^{k}}{\partial \bar{z}^{j}}-\frac{\sqrt{-1}}{2}\left(\frac{\partial \log h(z)}{\partial \bar{z}^{i}} \frac{\partial \varphi_{j}^{k}}{\partial \theta}-\frac{\partial \log h(z)}{\partial \bar{z}^{j}} \frac{\partial \varphi_{i}^{k}}{\partial \theta}\right)=0, & (1 \leq i, j, k \leq n) . \\
& (1 \leq .
\end{aligned}
$$

Lemma 3.3. For each $m \in Z$, the $\left\{\varphi_{\bar{j}, m}^{k}\right\} k, j=1, \cdots, n$ satisfy the following equation;

$$
\frac{\partial \varphi_{\bar{j}, m}^{k}}{\partial \bar{z}^{i}}-\frac{\partial \varphi_{\bar{i}, m}^{k}}{\partial \bar{z}^{j}}+\frac{m}{2}\left(\frac{\partial \log h}{\partial \bar{z}^{i}} \varphi_{\bar{j}, m}^{k}-\frac{\partial \log h}{\partial \bar{z}^{j}} \varphi_{\bar{i}, m}^{k}\right)=0 .
$$

Proof. This lemma is trivial from (3.7) and definitions of the $\varphi_{\bar{j}, m}^{k}$.

Q.E.D.

Moreover we have

Lemma 3.4. Let $\hat{\varphi}_{m}$ be the element of $\Gamma_{(C)}\left(\operatorname{Hom}\left({ }^{\circ} T^{\prime \prime}, T^{\prime}\right)\right)$ defined by

$$
\hat{\varphi}_{m}\left(Z_{\bar{j}}\right)=\sum_{k=1}^{n} e^{\sqrt{-1} m \theta} \varphi_{\bar{j}, m}^{k}(z) Z_{k} \quad(m \in Z) .
$$


Then there exists an element $\zeta_{m}$ of $\Gamma_{(C)}\left(T^{\prime}\right)$ for every non-zero integer $m$ such that

$$
\zeta_{m}=\sum_{k=1}^{n} \zeta_{m}^{k}(z) Z_{k}
$$

and

$$
\bar{\partial}_{b}^{(0)} \zeta_{m}\left(Z_{\bar{j}}\right)=\hat{\varphi}_{m}\left(Z_{\bar{j}}\right)+\sum_{k=1}^{n} \zeta_{m}^{k} \Phi_{k \bar{j}} \frac{\partial}{\partial \theta}
$$

Proof. Put $\psi_{\bar{j}, m}^{k}(z)=\varphi_{\bar{j}, m}^{k}(z) h^{m / 2}(z)$. Then we find from (2.2) and (3.6) that

$$
\psi_{\bar{j}, m}^{k}(z+\omega d)=\psi_{\bar{j}, m}^{k}(z) e^{-\sqrt{-1} m \arg f(z, d)} j(z, d)^{-m}=\psi_{\bar{j}, m}^{k}(z) f(z, d)^{-m},
$$

$\left(d \in Z^{2 n}\right)$.

Therefore if we set

$$
\psi_{m}^{k}=\sum_{j=1}^{n} \psi_{\bar{j}, m}^{k} d \bar{z}^{j},
$$

$\psi_{m}^{k}$ is regarded as the cross-section of the vector bundle $B^{-m} \otimes \bigwedge^{0,1}(T)$ over the abelian variety $T$, where $\wedge^{0,1}(T)$ represents the bundle consisting of $(0,1)$-type differential forms on $T$. Let $\bar{\partial}$ be the usual exterior derivation of type $(0,1)$ on $T$. Then it is clear that

$$
\bar{\partial} \psi_{m}^{k}=\sum_{i<j}\left(\frac{\partial \psi_{i}^{k}, m}{\partial \bar{z}^{i}}-\frac{\partial \psi_{\bar{i}, m}^{k}}{\partial \bar{z}^{j}}\right) d \bar{z}^{i} \wedge \bar{z}^{j}
$$

But

$$
\frac{\partial \psi_{\bar{j}, m}^{k}}{\partial \bar{z}^{i}}=\left[\frac{\partial \varphi_{\bar{j}, m}^{k}}{\partial \bar{z}^{i}}+\frac{m}{2} \varphi_{\bar{j}, m}^{k} \frac{\partial \log h}{\partial \bar{z}^{j}}\right] h^{m / 2},
$$

so that using (3.8), we obtain, for any $m \in Z$,

$$
\bar{\partial} \psi_{m}^{k}=0, \quad(k=1, \cdots, n)
$$

Now let $m \neq 0$. Then, since $B$ is a negative line bundle and the holomorphic tangent bundle of $T$ is analytically trivial $\left(\operatorname{dim}_{C} T \geqq 2\right)$, there is an element $\tilde{\eta}_{m}^{k}$ of $\Gamma\left(B^{-m}\right)$ such that, for any $m(\neq 0)$,

$$
\bar{\partial} \tilde{\eta}_{m}^{k}=\psi_{m}^{k}, \quad(k=1, \cdots, n) .
$$

If we write $\eta_{m}^{k}$ the pull-back of $\tilde{\eta}_{m}^{k}$ by the projection $\hat{\psi}: C^{n} \rightarrow T, \eta_{m}^{k}$ is the cross-section of the trivial bundle $C^{n} \times C$ over $C^{n}$ and satisfies the following relations; 


$$
\left\{\begin{array}{l}
\eta_{m}^{k}(\boldsymbol{z}+\omega \boldsymbol{d})=\eta_{m}^{k}(z) f(z, \boldsymbol{d})^{-m} \quad\left(\boldsymbol{d} \in Z^{2 n}\right) \\
\bar{\partial} \eta_{m}^{k}=\psi_{m}^{k}
\end{array}\right.
$$

Furthermore let $\zeta_{m}^{k}$ be the smooth function on $C^{n} \times S^{1}$ defined by

$$
\zeta_{m}^{k}(z, \theta)=e^{\sqrt{-1} m \theta} \eta_{m}^{k}(z) h^{-m / 2}(z) .
$$

We have then from (3.10)

$$
\zeta_{m}^{k}(z+\omega d, \theta+\arg f(z, d))=\zeta_{m}^{k}(z, \theta),
$$

that is, the vector field $\zeta_{m}$ defined by

$$
\zeta_{m}=\sum_{k=1}^{n} \zeta_{m}^{k} Z_{k}
$$

belongs to $\Gamma_{(C)}\left(T^{\prime}\right)$.

This $\zeta_{m}$ satisfies (3.9). Indeed it follows that

$$
\begin{aligned}
\left(\bar{\partial}_{b} \zeta_{m}\right)\left(Z_{j}\right) & =\sum_{k=1}^{n}\left(Z_{j} \zeta_{m}^{k}\right) Z_{k}+\sum_{k} \zeta_{m}^{k}\left[Z_{j}, Z_{k}\right] \\
& =\sum_{k} e^{\sqrt{-1} m \theta} \frac{\partial \eta_{m}^{k}}{\partial \bar{z}^{j}} h^{-m / 2} Z_{k}+\sum_{k} \zeta_{m}^{k} \Phi_{k j} \frac{\partial}{\partial \theta}, \quad(j=1, \cdots, n) .
\end{aligned}
$$

Here using $\bar{\partial} \eta_{m}^{k}=\psi_{m}^{k}$ in (3.10), we have

$$
\begin{aligned}
\left(\bar{\partial}_{b} \zeta_{m}\right)\left(Z_{\bar{j}}\right) & =\sum_{k} e^{\sqrt{-1} m \theta} \psi_{\bar{j}, m}^{k} h^{-m / 2} Z_{k}+\sum_{k} \zeta_{m}^{k} \Phi_{k j} \frac{\partial}{\partial \theta} \\
& =\sum_{k} e^{\sqrt{-1} m \theta} \varphi_{\bar{j}, m}^{k} Z_{k}+\sum_{k} \zeta_{m}^{k} \Phi_{k \bar{j}} .
\end{aligned}
$$

Next for (3.3), we set

$$
\left(\varsigma_{\varphi_{j}}\right)(z, \theta)=\sum_{m \in Z} \varphi_{j, m}(z) e^{\sqrt{-1} m \theta}, \quad(j=1, \cdots, n) .
$$

Let $\hat{\varphi}_{m} \in \Gamma_{(C)}\left(\operatorname{Hom}\left({ }^{\circ} T^{\prime \prime}, T^{\prime}\right)\right)$ be as in Lemma 3.4. If we define the element $\varphi_{m} \in \Gamma_{(C)}\left(\operatorname{Hom}\left({ }^{\circ} T^{\prime \prime}, T^{\prime}\right)\right)$ for each $m \in Z$, by

$$
\varphi_{m}\left(Z_{j}\right)=\hat{\varphi}_{m}\left(Z_{j}\right)+\varphi_{j, m} e^{\sqrt{-1} m \theta} \frac{\partial}{\partial \theta}, \quad(j=1, \cdots, n),
$$

then each $\varphi_{m}$ is the $e^{\sqrt{-1} m \theta-c o m p o n e n t ~ o f ~ t h e ~ F o u r i e r ~ e x p a n s i o n ~} \subseteq(\varphi)$ of $\varphi$, that is,

$$
S(\varphi)=\sum_{m \in Z} \varphi_{m}
$$


Here $\subseteq(\varphi)$ is defined by

$$
\mathfrak{S}(\varphi)\left(Z_{\bar{j}}\right)=\sum_{k} \mathfrak{S}\left(\varphi_{\mathrm{j}}^{k}\right) Z_{k}+\mathfrak{S}\left(\varphi_{\mathrm{j}}\right) \frac{\partial}{\partial \theta}, \quad(j=1, \cdots, n) .
$$

Since $\bar{\partial}_{b}^{(1)} \varphi=0$, we find

$$
\bar{\partial}_{b}^{(1)} \varphi_{m}=0, \quad \text { for every } m \in Z \text {. }
$$

Moreover we can write, for any non-zero integer $m$, using $\zeta_{m}$ in Lemma 3.4,

$$
\left(\varphi_{m}-\bar{\partial}_{b}^{(0)} \zeta_{m}\right)\left(Z_{j}\right)=\tilde{\varphi}_{j, m}(z) e^{\sqrt{-1} m \theta} \frac{\partial}{\partial \theta}, \quad j=1, \cdots, n,
$$

where the $\tilde{\varphi}_{j, m}(z)$ are smooth functions on $C^{n}$ such that

$$
\tilde{\varphi}_{j, m}(z+\omega d)=\tilde{\varphi}_{j, m}(z), \quad \text { for all }(z, d) \in C^{n} \times Z^{2 n} \text {. }
$$

Lemma 3.5. Let $m$ by any non-zero integer. Then $\varphi_{m}-\bar{\partial}_{b}^{(0)} \zeta_{m}$ in (3.11) is $\bar{\partial}_{b}^{(0)}$-boundary, i.e., there exists an element $\eta_{m} \frac{\partial}{\partial \theta}$ of $\Gamma_{(C)}\left(T^{\prime}\right)$ such that

$$
\bar{\partial}_{b}^{(0)}\left(\eta_{m} \frac{\partial}{\partial \theta}\right)=\varphi_{m}-\bar{\partial}_{b}^{(0)} \zeta_{m}
$$

Proof. As $\bar{\partial}_{b}^{(1)}\left(\varphi_{m}-\bar{\partial}_{b}^{(0)} \zeta_{m}\right)=0$, the family of functions $\left\{\tilde{\varphi}_{j, m}\right\}_{j=1, \ldots, n}$ in the right hand side of (3.11) satisfies the following relations;

$$
\begin{aligned}
\frac{\partial \tilde{\varphi}_{j, m}}{\partial \bar{z}^{i}}-\frac{\partial \tilde{\varphi}_{i, m}}{\partial \bar{z}^{j}}+\frac{m}{2}\left(\frac{\partial \log h}{\partial \bar{z}^{i}} \tilde{\varphi}_{j, m}-\frac{\partial \log h}{\partial \bar{z}^{j}} \tilde{\varphi}_{i, m}\right)= & , \\
& 1 \leq 1, j \leq n .
\end{aligned}
$$

Therefore this lemma is proved in the same way as Lemma 3.4. Q.E.D.

We obtain from Lemma 3.5 the following

Proposition 3.6. Let $\varphi$ be an arbitrary element of $\Gamma_{(C)}\left(\operatorname{Hom}\left({ }^{\circ} T^{\prime \prime}, T^{\prime}\right)\right)$ such that $\bar{\partial}_{b}^{(1)} \varphi=0$. Moreover let $\mathfrak{S}(\varphi)$ be the Fourier expansion of $\varphi$ with respect to the parameter $\theta$ of $S^{1}$;

$$
\Im(\varphi)=\sum_{m \in Z} \varphi_{m}
$$

where the $\varphi_{m}$ are elements of $\Gamma_{(C)}\left(\operatorname{Hom}\left({ }^{\circ} T^{\prime \prime}, T^{\prime}\right)\right)$ defined by

$$
\varphi_{m}\left(Z_{\bar{j}}\right)=e^{\sqrt{-1} m \theta}\left(\sum_{k} \varphi_{\bar{j}, m}^{k}(z) Z_{k}+\varphi_{j, m}(z) \frac{\partial}{\partial \theta}\right) .
$$

Then for all $m(\neq 0) \in Z$, we have, 


$$
\varphi_{m}=\bar{\partial}_{b}^{(0)} \zeta_{m}, \quad \text { for some } \zeta_{m} \in \Gamma_{(C)}\left(T^{\prime}\right) .
$$

Furthermore we can prove the following

Proposition 3.7. Let all notations be as in the above proposition. Let $\varphi \in \Gamma_{(C)}\left(\operatorname{Hom}\left({ }^{\circ} T^{\prime \prime}, T^{\prime}\right)\right.$, with $\bar{\partial}_{b}^{(1)} \varphi=0$. Then $\varphi$ is $\bar{\partial}_{b}$-cohomologous to $\varphi_{0}$, where $\widetilde{S}(\varphi)=\varphi_{0}-\sum_{z-(0) \ni m} \varphi_{m}$.

Proof. In general let $f$ be any $C^{\infty}$-function on $C^{n} \times S^{1}$. Then $\mathfrak{S}(f)$ converges uniformly on every compact subset of $C^{n} \times S^{1}$. Now for any $\varphi \in \Gamma_{(C)}\left(\right.$ Hom $\left.\left({ }^{\circ} T^{\prime \prime}, T^{\prime}\right)\right)$, we define the norm, denoted by $|\varphi|$, as follows; Let $\varphi\left(Z_{j}\right)=\sum \varphi_{j}^{k} Z_{k}-\varphi_{j} \frac{\partial}{\partial \theta}$. Then we have

$$
|\varphi|=\max _{1 \leq j, k \leq n}\left\{\sup \left|\varphi_{\bar{j}}^{k}\right|, \sup \left|\varphi_{j}\right|\right\} .
$$

This is well-defined because of the condition (C). Here if we put $\mathfrak{S}_{k}(\varphi)=\sum_{|m| \leq k} \varphi_{m}$, for any non-negative integer $k$, we find that for any $\varepsilon>0$, there exists an integer $k(\varepsilon) \geqq 0$, such that

$$
\left|\varphi-\mathfrak{S}_{k(\varepsilon)}(\varphi)\right|<\varepsilon .
$$

Let $\tau$ be the isomorphism of the complex $\left\{\Gamma\left(\bigwedge^{k}\left({ }^{\circ} T^{\prime \prime}\left(B_{1}\right)\right)^{*} \otimes T^{\prime}\left(B_{1}\right)\right)\right.$, $\left.\bar{\partial}_{b}^{k}\right\}$ onto $\left\{\Gamma_{(C)}\left(\bigwedge^{k}\left({ }^{\circ} T^{\prime \prime}\right)^{*} \otimes T^{\prime}\right), \bar{\partial}_{b}(k)\right\}$ as in the proof of Proposition 3.1.

On the other hand we impose the hermitian innerproduct, $\langle$,$\rangle on$ $C T\left(B_{1}\right)$ such that, $\psi_{*}\left(Z_{\overline{1}}\right), \cdots, \psi_{*}\left(Z_{n}\right), \psi_{*}\left(Z_{1}\right), \cdots, \psi^{*}\left(Z_{n}\right)$ and $\psi_{*}(\partial / \partial \theta)$ are orthonormal basis. For every $\tilde{\varphi} \in \Gamma\left(\bigwedge^{*}\left({ }^{\circ} T\left(B_{1}\right)^{*} \otimes T^{\prime}\left(B_{1}\right)\right)\right.$, we set

$$
\|\tilde{\varphi}\|=\sum_{1 \leq j_{1}<\cdots j_{k} \leq n}\left\langle\tilde{\varphi}\left(\psi_{*}\left(Z_{j_{1}}\right), \cdots, \psi_{*}\left(Z_{j_{k}}\right), \tilde{\varphi}\left(\psi_{*}\left(Z_{j_{1}}\right), \cdots, \psi_{*}\left(Z_{\tilde{j}}{ }^{k}\right)\right)\right\rangle \frac{1}{2} .\right.
$$

The $L_{2}$-norm, denoted by \|\|$_{B_{1}}$, on $\Gamma\left(\bigwedge^{k}\left({ }^{\circ} T^{\prime \prime}\left(B_{1}\right)\right)^{*} \otimes T^{\prime}\left(B_{1}\right)\right)$ is defined by

$$
\|\tilde{\varphi}\|_{B_{1}}=\int_{B_{1}}\|\tilde{\varphi}\| d v
$$

where $d v$ denotes the volume element associated with the hermitian inner product $\langle$,$\rangle on C T\left(B_{1}\right)$.

We can further form the formal adjoint

$$
\bar{\partial}_{2}^{(k) *}: \Gamma\left(\bigwedge^{k}\left({ }^{\circ} T^{\prime \prime}\left(B_{1}\right)\right)^{*} \otimes T^{\prime}\left(B_{1}\right)\right) \rightarrow \Gamma\left(\wedge^{k-1}\left({ }^{\circ} T^{\prime \prime}\left(B_{1}\right)\right)^{*} \otimes T^{\prime}\left(B_{1}\right)\right)
$$

of $\bar{\partial}_{b}^{(k-1)}$ with respect to the above norm \|\|$_{B_{1}},(k=1, \cdots, n)$.

Now take an element $\varphi$ of $\Gamma_{(C)}\left(\left({ }^{\circ} T^{\prime \prime}\right) T^{\prime}\right)$ with $\bar{\partial}_{\beta}^{(1)} \varphi=0$. For any $\varepsilon>0$. there exirts an integer $k(\varepsilon)$ such that 


$$
\left\|\tau^{-1} \varphi-\tau^{-1}\left(\Im_{k(s)}(\varphi)\right)\right\|<\varepsilon .
$$

Moreover it is clear that $\bar{\partial}_{b}^{(1)} \tau^{-1} \varphi=0$. Since ${ }^{\circ} T^{\prime \prime}\left(B_{1}\right)$ is strongly pseudoconvex and $\operatorname{dim}_{R} B_{1} \geqq 5$, we know from $\S 6$ [1] that there exists $\tilde{\zeta} \in \Gamma\left(T^{\prime}\left(B_{1}\right)\right)$ and $\tilde{\eta} \in \Gamma\left({ }^{\circ} T^{\prime \prime}\left(B_{1}\right)^{*} \otimes T^{\prime}\left(B_{1}\right)\right)$ such that

$$
\tau^{-1} \varphi-\tau^{-1} \varphi_{0}=\tilde{\eta}+\bar{\partial}_{b}^{(0)} \tilde{\zeta},
$$

where $\left(\bar{\partial}_{b}^{(2) *} \cdot \bar{\partial}_{b}^{(1)}+\bar{\partial}_{b}^{(0)} \bar{\partial}_{b}^{(1) *}\right) \tilde{\eta}=0$.

We shall show $\tilde{\eta}=0$. Indeed suppose $\tilde{\eta} \neq 0$. Then it follows that

$$
\left\|\tau^{-1} \varphi-\tau^{-1} \varphi_{0}\right\|_{B_{1}} \geqq\|\tilde{\eta}\|_{B_{1}}>\varepsilon_{1}, \quad \text { for some } \varepsilon_{1}>0 \text {. }
$$

Here for any $\varepsilon>0$ with $\varepsilon<\varepsilon_{1}$, we choose an integer $k(\varepsilon)$ satisfying (3.12), and put

$$
\widetilde{S}_{k(s)}^{\prime}(\varphi)=\mathbb{S}_{k(s)}(\varphi)-\varphi_{0} .
$$

It follows from Proposition 3.6 that there is an element $\varepsilon \in \Gamma\left(T^{\prime}\right)$ such that

$$
\widetilde{S}_{k(\varepsilon)}^{\prime}(\varphi)=\bar{\partial}_{b}^{(0)} \zeta
$$

so that we have

$$
\varepsilon>\left\|\tau^{-1} \varphi-\tau^{-1} \varphi_{0}-\tau^{-1} \widetilde{S}_{k(s)}^{\prime}(\varphi)\right\|_{B_{1}}=\left\|\tau^{-1} \varphi-\tau^{-1} \varphi_{0} \bar{\partial}_{b}^{(0)} \tau^{-1} \zeta\right\|_{B_{1}} \geqq\|\tilde{\eta}\|_{B_{1}} .
$$

This is a contradiction and so our proposition is proved.

Q.E.D.

By virtue of the above arguments, in order to determine the infinitesimal deformation $H_{(G)}^{1}\left({ }^{\circ} T^{\prime \prime}\right)\left(\cong H^{1}\left({ }^{\circ} T^{\prime \prime}\left(B_{1}\right)\right)\right.$ ), it is enough to consider the following subcomplex (3.13) of $\left\{\Gamma_{(C)}\left(\wedge^{p}\left({ }^{\circ} T^{\prime \prime}\right)^{*} \otimes T^{\prime}\right), \bar{\partial}_{b}^{, p)}\right\}$; Let

$$
\varphi \in \Gamma\left(\wedge^{k}\left({ }^{\circ} T^{\prime \prime}\right)^{*} \otimes T^{\prime}\right)
$$

and set

$$
\varphi\left(Z_{\bar{j}_{1}}, \cdots, Z_{\bar{j}_{k}}\right)=\sum_{i=1}^{n} \varphi_{\bar{j}_{1}, \cdots, \bar{j}_{k}}^{i} Z_{i}+\varphi_{j_{1}, \cdots j_{k}} \frac{\partial}{\partial \theta} \quad\left(1 \leq j_{1}, \cdots, j_{k} \leqq n\right) .
$$

We denote by $\Gamma_{r}\left(\bigwedge^{k}\left({ }^{\circ} T^{\prime \prime}\right)^{*} \otimes T^{\prime}\right)$ the set of all $\varphi \in \Gamma\left(\bigwedge^{k}\left({ }^{\circ} T^{\prime \prime}\right)^{*} \otimes T^{\prime}\right)$ such that $\varphi_{\bar{j}_{1}, \ldots, \bar{j}_{k}}^{i}$ and $\varphi_{j_{1}, \cdots, j_{k}}$ are smooth functions on the abelian variety $T$. Then the complex

$$
\begin{aligned}
0 \longrightarrow & \Gamma_{T}\left(T^{\prime}\right) \stackrel{\bar{\partial}_{b}^{(0)}}{\longrightarrow} \Gamma_{T}\left(\left({ }^{\circ} T^{\prime \prime}\right) * \otimes T^{\prime}\right) \\
& \stackrel{\bar{\partial}_{b}^{(1)}}{\longrightarrow} \Gamma_{T}\left(\bigwedge^{2}\left({ }^{\circ} T^{\prime \prime}\right)^{*} \otimes T^{\prime}\right) \stackrel{\bar{\partial}_{b}^{(2)}}{\longrightarrow} \cdots,
\end{aligned}
$$


is the required one.

THEOREM 3.8. Let ${ }^{\circ} T^{\prime \prime}$ be the strongly pseudo-convex CR-structure on $C^{n} \times S^{1}(n \geqq 2)$ induced from the negative line bundle $B$ over the abelian variety $T\left(\operatorname{dim}_{C} T=n\right)$, as before. Let $\varphi \in \Gamma_{(C)}\left(\left({ }^{\circ} T^{\prime \prime}\right)^{*} \otimes T^{\prime}\right)$ with

$$
\varphi\left(Z_{\bar{j}}\right)=\sum_{k=1}^{n} C_{\bar{j}}^{k} Z_{k}+a_{j} \frac{\partial}{\partial \theta}, \quad(j=1, \cdots, n),
$$

where the $C_{\bar{j}}^{k}$ and the $a_{j}$ are arbitrary constants such that

$$
\sum_{k=1}^{n} C_{j}^{k} Q_{k \bar{i}}=\sum_{k=1}^{n} C_{i}^{k} Q_{k \bar{j}}, \quad(1 \leq i, j \leq n)
$$

Then any element of $H_{(C)}^{1}\left({ }^{\circ} T^{\prime \prime}\right)$ can be represented by some element $\rho$ as above.

Proof. Take $\varphi \in \Gamma_{T}\left(\left(^{\circ} T^{\prime \prime}\right)^{*} \otimes T^{\prime}\right)$ such that $\bar{\partial}_{b}^{(1)} \varphi=0$ and

$$
\varphi\left(Z_{\bar{j}}\right)=\sum_{k=1}^{n} \varphi_{\bar{j}}^{k} Z_{k}+\varphi_{j} \frac{\partial}{\partial \theta}, \quad(j=1, \cdots, n) .
$$

Recall that $\varphi_{j}^{k}$ and $\varphi_{j}$ are $C^{\infty}$-functions on $T$. By Proposition 3.2, $\bar{\partial}_{0}^{(1)} \varphi=0$ means that $\varphi$ satisfies equations (3.2) and (3.3). Here using $\varphi_{j}^{k}$ and $\varphi_{j}$ in (3.16), we put

$$
\psi=\sum_{j, k=1}^{n} \varphi_{\bar{j}}^{k} \frac{\partial}{\partial z^{k}} \otimes d \bar{z}^{j}, \quad \omega=\sum_{j=1}^{n} \varphi_{j} d \bar{z}^{j}
$$

and

$$
\Phi=\sum_{i, j=1}^{n} \Phi_{i \bar{j}} d z^{j} \wedge d \bar{z}^{j}(=\sqrt{-1} \partial \bar{\partial} \log h), \quad \text { (c.f. (1.2)) }
$$

where $\Phi_{i \bar{j}}=2 \pi Q_{i \bar{j}}$.

Let $\wedge^{(p, q)}(T)$ be the set of all differential forms of type $(p, q)$ on $T$. Moreover let $\pi$ be the generalized interior product, that is, for any $\tilde{\psi} \in \bigwedge^{(p, q)}(T) \otimes \Gamma(T(T))$ with

$$
\begin{aligned}
\tilde{\psi}=\sum_{j_{1}, \cdots, j_{q}} \sum_{i} \cdots \sum_{i_{p}} \sum_{k} A_{i_{1}, \cdots, i_{p}, \bar{j}_{1}, \cdots, \bar{j}_{q}}^{k} d z^{i_{1}} \wedge \cdots \wedge d z^{i_{p}} \wedge d \bar{z}^{j_{1}} \wedge \\
\cdots \wedge d \bar{z}^{j_{q}} \otimes \frac{\partial}{\partial z^{k}}
\end{aligned}
$$

the linear map $\tilde{\psi} \pi: \bigwedge^{(\ell, m)}(T) \rightarrow \bigwedge^{(\ell+p-1, m+q)}(T)$ is defined by 


$$
\begin{aligned}
\tilde{\psi} \pi( & \left.\sum_{\substack{\alpha_{1}, \ldots, \alpha_{\ell} \\
\beta_{1}, \cdots, \beta_{m}}} B_{\alpha_{1}, \cdots, \alpha_{\ell}, \bar{\beta}_{1}, \cdots, \beta_{m}} d z^{\alpha_{1}} \wedge \cdots \wedge d z^{\alpha_{\ell}} \wedge d \bar{z}^{\beta_{1}} \wedge \cdots d \bar{z}^{\beta_{m}}\right) \\
= & \sum_{i_{1}, \cdots, i_{p}} \sum_{j_{1}, \ldots, j_{q} \alpha_{1}, \cdots, \alpha_{\ell} \beta_{1}, \cdots, \beta_{m}} \sum_{k}\left\{\sum_{\ell^{\prime}=1}^{\ell}(-1)^{\ell^{\prime}+1} A_{i_{1}, \cdots, i_{p}, \bar{j}_{q}}^{k} d z^{i_{1}} \wedge\right. \\
& \cdots \wedge d z^{i_{p}} \wedge d \bar{z}^{j_{1}} \wedge \cdots \wedge d \bar{z}^{j_{q}} \wedge B_{\alpha_{1}, \cdots, \alpha_{\ell} \beta_{1}, \cdots, \beta_{m}} d z^{\alpha_{1}} \wedge \\
& \left.\cdots \wedge d z^{\alpha_{\ell^{\prime}-1}} \wedge\left\langle\frac{\partial}{\partial z^{k}} d z^{\alpha_{\ell^{\prime}}}\right\rangle \wedge \cdots \wedge d z^{\alpha_{\ell}} \wedge d \bar{z}^{\beta_{1}} \wedge \cdots \wedge d \bar{z}^{\beta_{m}}\right\} .
\end{aligned}
$$

Then (3.2) and (3.3) are rewritten as follows;

$$
\bar{\partial} \psi=0,
$$

and

$$
\psi \pi \Phi-\bar{\partial} \omega=0 \text {. }
$$

Next we examine conditions for $\bar{\partial}_{b}$-boundary in the complex (3.13). For this aim let

$$
\psi=\sum_{j=1}^{n} \psi^{j} Z_{j}+\eta \frac{\partial}{\partial \theta}
$$

be an element of $\Gamma_{T}\left(T^{\prime}\right)$ such that $\varphi=\bar{\partial}_{b}^{(0)} \psi$. Then we obtain

$$
\left\{\begin{array}{l}
\varphi_{j}^{k}=\frac{\partial \psi^{k}}{\partial \bar{z}^{j}} \quad(1 \leq j, k \leq n), \\
\varphi_{j}=\sum_{k=1}^{n} \psi^{k} \Phi_{k \bar{j}}+\frac{\partial \eta}{\partial \bar{z}^{j}}, \quad(j=1, \cdots, n) .
\end{array}\right.
$$

Set here

$$
\xi=\sum_{k=1}^{n} \psi^{k} \frac{\partial}{\partial z^{k}}
$$

Then (3.19) means that

$$
\psi=\bar{\partial} \xi \text { and } \omega=\xi \pi \Phi+\bar{\partial} \eta \text {. }
$$

Therefore in terms of (3.17), (3.18) and (3.20) the complex (3.13) reduces to the following one;

$$
\begin{aligned}
& 0 \underset{\longrightarrow}{\longrightarrow} \Gamma(T(T)) \oplus C^{\infty}(T) \stackrel{\bar{\partial}_{\phi}^{(0)}}{\longrightarrow}\left(\bigwedge^{(0,1)}(T) \otimes \Gamma(T(T))\right) \oplus \bigwedge^{(0,1)}(T) \\
& \stackrel{\bar{\partial}_{\phi}^{(1)}}{\longrightarrow}\left(\bigwedge^{(0,2)}(T) \otimes \Gamma(T(T))\right) \oplus \bigwedge^{(0,2)}(T),
\end{aligned}
$$

where 


$$
\bar{\partial}_{\Phi}^{(0)}(\xi, \eta)=(\bar{\partial} \xi, \xi \pi \Phi+\bar{\partial} \eta)
$$

and

$$
\bar{\partial}_{\Phi}^{(1)}\left(\psi^{\prime}, \omega^{\prime}\right)=\left(\bar{\partial} \psi^{\prime}, \psi^{\prime} \pi \Phi-\bar{\partial} \omega^{\prime}\right),
$$

for $(\xi, \eta) \in \Gamma(T(T)) \oplus C^{\infty}(T)$ and $\left(\psi^{\prime}, \omega^{\prime}\right) \in\left(\bigwedge^{(0,1)}(T) \otimes T(T)\right) \oplus \bigwedge^{(0,1)}(T)$.

Now let $\left(\psi^{\prime}, \omega^{\prime}\right) \in \operatorname{Ker} \bar{\partial}_{\phi}^{(1)}$. As $\bar{\partial} \psi^{\prime}=0$, it follows that there exist constants $\left\{C_{\bar{j}}^{k}\right\} 1 \leq j, k \leq n$ and $\xi \in \Gamma(T(T))$ such that

$$
\psi^{\prime}=\sum_{j, k=1}^{n} C_{\bar{j}}^{k} d \bar{z}^{j} \otimes \frac{\bar{\partial}}{\partial z^{k}}+\bar{\partial} \xi
$$

Moreover we have

$$
\psi^{\prime} \pi \Phi-\bar{\partial} \omega^{\prime}=\sum_{i, j, k} C_{\bar{j}}^{k} \Phi_{k \bar{i}} d \bar{z}^{j} \wedge d \bar{z}^{i}+\bar{\partial}\left(\xi \pi \Phi-\omega^{\prime}\right)=0
$$

Therefore the $\bar{\partial}$-cohomology class of $\sum_{i, j, k} C_{\bar{j}}^{k} \Phi_{k \bar{i}} d \bar{z}^{j} \wedge d \bar{z}^{i}$ is zero. However since $C_{\bar{j}}^{k}$ and $\Phi_{k \bar{j}}$ are constants, we get

$$
\sum_{i, j, k} C_{\bar{j}}^{k} \Phi_{k \bar{i}} d \bar{z}^{j} \wedge d \bar{z}^{i}=0
$$

This fact shows (3.15). Moreover it follows from (3.21) and (3.22) that $\bar{\partial}\left(\xi \pi \Phi-\omega^{\prime}\right)=0$, so that we are able to write

$$
\xi \pi \Phi-\omega^{\prime}=\sum_{j=1}^{n} a_{j} d \bar{z}^{j}+\bar{\partial} \eta_{0}
$$

where the $a_{j}$ are constants and $\eta_{0}$ is a smooth function on $T$. Clearly

$$
\left(\sum_{j, k} C_{\bar{j}}^{k} \frac{\partial}{\partial z^{k}} \otimes d \bar{z}^{j}, \sum_{j} a_{j} d \bar{z}^{j}\right)
$$

belongs to $\operatorname{Ker} \bar{\partial}_{\phi}^{(1)}$. It follows that $\left(\psi^{\prime}, \omega^{\prime}\right)$ and

$$
\left(\sum_{j, k} C_{\bar{j}}^{\dot{k}} \frac{\partial}{\partial z^{k}} \otimes d \bar{z}^{j}, \sum_{j} a_{j} d \bar{z}^{j}\right)
$$

are $\bar{\partial}_{\phi}^{(0)}$-cohomologous. Indeed we have

$$
\begin{array}{r}
\left(\psi^{\prime}, \omega^{\prime}\right)-\left(\sum_{j, k} C_{\bar{j}}^{k} \frac{\partial}{\partial z^{k}} \otimes d \bar{z}^{j}, \sum_{j} a_{j} d \bar{z}^{j}\right) \\
=\left(\bar{\partial} \xi, \xi \pi \Phi-\bar{\partial} \eta_{0}\right)=\bar{\partial}_{\Phi}^{(0)}\left(\xi,-\eta_{0}\right) .
\end{array}
$$

Thus our theorem is completely proved.

Q.E.D. 


\section{§4. Integrable $C R$-structures on $C^{n} \times S^{1}$.}

Let all notations be as before. Recall that ${ }^{\circ} T^{\prime \prime}$ is the strongly pseudoconvex $C R$-structure on $C^{n} \times S^{1}$ induced by $\psi: C^{n} \times S^{1} \rightarrow C^{n} \times C$ (see the bottom of Proposition 2.1). Let now $\varphi \in \Gamma_{(C)}\left(\left({ }^{\circ} T^{\prime \prime}\right)^{*} \otimes T^{\prime}\right)$ be at finite distance from ${ }^{\circ} T^{\prime \prime}$. Then ${ }^{\varphi} T^{\prime \prime}=\left\{X-\varphi(X) ; X \in{ }^{\circ} T^{\prime \prime}\right\}$ becomes an almost $C R$-structure on $C^{n} \times S^{1}$. It follows from Proposition 1.4 that $\varphi$ is integrable if and only if $P(\varphi)=0$, where the linear map $P: \Gamma\left(\left(^{\circ} T^{\prime \prime}\right)^{*} \otimes\right.$ $\left.T^{\prime}\right) \rightarrow \Gamma\left(\wedge^{2}\left({ }^{\circ} T^{\prime \prime}\right)^{*} \otimes T^{\prime}\right)$ is defined by (1.6).

Proposition 4.1. Let $\varphi \in \Gamma_{(C)}\left(\left(^{\circ} T^{\prime \prime}\right)^{*} \otimes T^{\prime}\right)$ be as in Theorem 3.8. Then $\varphi$ is integrable.

Proof. This is trivial from the definition of $P$ and (3.14). Q.E.D.

For convenience sake we write $\mathscr{H}^{1}$ the set of all $\varphi \in \Gamma_{(C)}\left(\left({ }^{\circ} T^{\prime \prime}\right)^{*} \otimes T^{\prime}\right)$ satisfying (3.14) and (3.15) in Theorem 3.8 , so that $\mathscr{H}^{1}$ is isomorphic to $H_{(C)}^{1}\left({ }^{\circ} T^{\prime \prime}\right)$. Let $\varphi$ be any element of $\mathscr{H}^{1}$ with

$$
\varphi\left(Z_{\bar{j}}\right)=\sum_{k=1}^{n} C_{\bar{j}}^{k} Z_{k}-\frac{\sqrt{-1}}{2} a_{j} \frac{\partial}{\partial \theta}, \quad(j=1, \cdots, n),
$$

where the norm $|\varphi|$ of $\varphi$ is sufficiently small. Then $\varphi$ is at finite distance to ${ }^{\circ} T^{\prime \prime}$ and we get

$$
\begin{aligned}
& Z_{\bar{j}}-\varphi\left(Z_{\bar{j}}\right)=\left(\frac{\partial}{\partial \bar{z}^{j}}-\sum_{k=1}^{k} C_{\bar{j}}^{k} \frac{\partial}{\partial z^{k}}\right) \\
&-\frac{\sqrt{-1}}{2}\left(\frac{\partial \log h}{\partial \bar{z}^{j}}+\sum_{k=1}^{n} C_{j}^{k} \frac{\partial \log h}{\partial z^{k}}-a_{j}\right) \frac{\partial}{\partial \theta}, \\
& j=1, \cdots, n .
\end{aligned}
$$

And the $C R$-structure $\varphi T^{\prime \prime}$ is generated by $\left\{Z_{j}-\varphi\left(Z_{j}\right)\right\} j=1, \cdots, n$.

We shall next determine complex structures on $C^{n} \times C$ which induce $C R$-structures $\varphi \in \mathscr{H}^{1}$ on $C^{n} \times S^{1}$ by the map $\psi: C^{n} \times S^{1} \rightarrow C^{n} \times C$. Remember that $\psi$ is defined by

$$
\psi(z, \theta)=\left(z, \frac{e^{\sqrt{-1} \theta}}{\sqrt{h(z)}}\right) \quad \text { for }(z, \theta) \in C^{n} \times S^{1}
$$

Proposition 4.2. Let $\varphi \in \mathscr{H}^{1}$ with (4.1). Let $z^{1}, \cdots, z^{n}$ and $\zeta$ be the canonical coordinates of $\boldsymbol{C}^{n} \times \boldsymbol{C}$, and put 


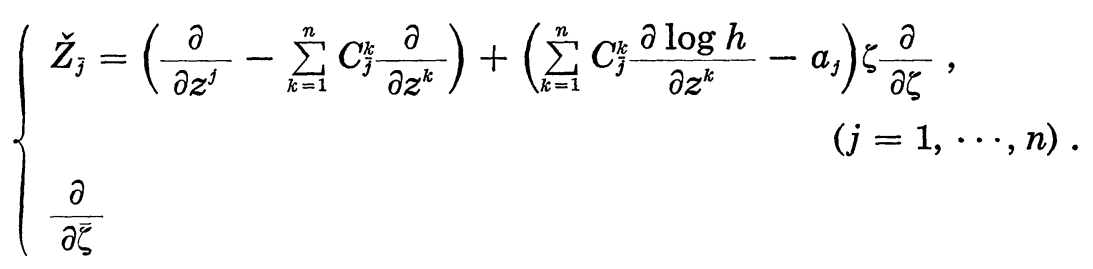

Then the CR-structure " $T^{\prime \prime}$ on $C^{n} \times S^{1}$ is the one which is induced from the complex structure $\left\{\left\{\check{Z}_{\overline{1}}^{\varphi}, \cdots, \check{Z}_{n}^{\varphi}, \partial / \partial \bar{\zeta}\right\}\right\}$ on $C^{n} \times C$ by $\psi$, that is,

$$
\psi_{*}\left(\varphi T^{\prime \prime}\right)=\psi_{*}\left(C T\left(C^{n} \times S^{1}\right)\right) \cap\left\{\left\{\check{Z}_{\overline{1}}^{\varphi}, \cdots, \check{Z}_{\bar{\varphi}}^{\varphi}, \frac{\partial}{\partial \bar{\zeta}}\right\}\right\} .
$$

Proof. First of all we see the system (4.3) is integrable. In fact it follows that

$$
\begin{aligned}
{\left[\check{Z}_{\bar{i}}^{\varphi}, \check{Z}_{\bar{j}}^{\varphi}\right] } & =-\sum_{k=1}^{n}\left(C_{\bar{i}}^{k} \frac{\partial^{2} \log h}{\partial \bar{z}^{j} \partial z^{k}}-C_{\bar{j}}^{k} \frac{\partial^{2} \log h}{\partial \bar{z}^{i} \partial z^{k}}\right) \zeta \frac{\partial}{\partial \zeta} \\
& =-\sqrt{-1} \sum_{k=1}^{n}\left(C_{\bar{i}}^{k} Q_{k \bar{j}}-C_{\bar{j}}^{k} Q_{k \bar{i}}\right) \zeta \frac{\partial}{\partial \zeta}=0,
\end{aligned}
$$

and

$$
\left[\check{Z}_{\bar{\jmath}}^{\varphi}, \frac{\partial}{\partial \bar{\zeta}}\right]=0, \quad(1 \geq i, j \leq n)
$$

Thus (4.3) is integrable. We next show the relation (4.4). For this aim it is enough to prove that

$$
\psi_{*}\left({ }^{\varphi} T^{\prime \prime}\right) \subset\left\{\left\{\check{Z}_{\overline{1}}^{\varphi}, \cdots, \check{Z}_{n}^{\varphi}, \frac{\partial}{\partial \bar{\zeta}}\right\}\right\}
$$

However it follows that for all $(z, \theta) \in C^{n} \times S^{1}$,

$$
\begin{gathered}
\psi_{*(z, \theta)}\left(Z_{\bar{j}}-\varphi\left(Z_{\bar{j}}\right)\right) \\
=\left(\frac{\partial}{\partial \bar{z}^{j}}-\sum_{k} C_{\bar{j}}^{k} \frac{\partial}{\partial z^{k}}\right)_{\Psi(z, \theta)}+\left\{\left(\sum_{k} C_{\bar{j}}^{k} \frac{\partial \log h}{\partial z^{k}}-a_{j}\right) \zeta \frac{\partial}{\partial \zeta}\right\}_{\Psi(z, \theta)} \\
-\left\{\left(\frac{1}{2} \frac{\partial \log h}{\partial z^{j}}-\sum_{k} C_{\bar{j}}^{k} \frac{\partial \log h}{\partial z^{k}}-a_{j}\right) \bar{\zeta} \frac{\partial}{\partial \zeta}\right\}_{\psi(z, \theta)}, \\
(j=1, \cdots, n) . \quad \text { Q.E.D. }
\end{gathered}
$$

Now for any $\varphi \in \mathscr{H}^{1}$ with (4.1), we denote by $T_{\varphi}^{\prime \prime}\left(C^{n} \times C\right)$ the subbundle of $C T\left(C^{n} \times C\right)$ generated by the system (4.3) in Proposition 4.2, or the complex structure on $C^{n} \times C$ defined by (4.3). Moreover let $T^{\varphi}$ be the diffeomorphism of $C^{n} \times C$ onto $C^{n} \times C$ defined by 


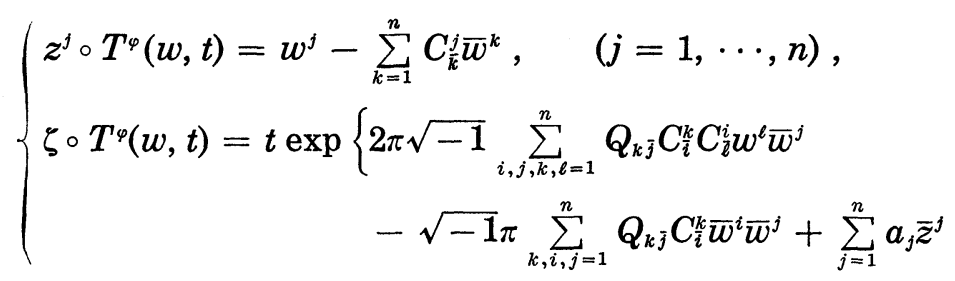

where $(w, t)$ and $(z, \zeta)$ are usual coordinates on $\boldsymbol{C}^{n} \times \boldsymbol{C}$.

Then we have the following

Proposition 4.3. Let $\varphi$ be an element of $\mathscr{H}^{1}$ with (4.1). Then the complex structure $T_{\varphi}^{\prime \prime}\left(C^{n} \times C\right)$ is induced from the standard complex structure on $C^{n} \times C$ by the diffeomorphism $T^{\varphi}: C^{n} \times C \rightarrow C^{n} \times C$ defined by (4.5), that is,

$$
T^{\prime \prime} \varphi\left(C^{n} \times C\right)=\left\{\left\{T_{*}^{\varphi}\left(\frac{\partial}{\partial \bar{w}^{1}}\right), \cdots, T_{*}^{\varphi}\left(\frac{\partial}{\partial \bar{w}^{n}}\right), T_{*}^{\varphi}\left(\frac{\partial}{\partial \bar{t}}\right)\right\}\right\}
$$

Proof. By direct calculations we have

$$
\begin{aligned}
T_{*}^{\varphi}\left(\frac{\partial}{\partial \bar{w}^{j}}\right)= & \left(\frac{\partial}{\partial \bar{z}^{j}}-\sum_{k=1}^{n} C_{j}^{k} \frac{\partial}{\partial z^{k}}\right) \\
& -\left\{-2 \pi \sqrt{-1} \sum_{i, k=1}^{n} Q_{k j} C_{\ell}^{k}\left(\bar{z}^{i} T \varphi\right)-a_{j}\right\} \zeta \circ T^{\varphi} \frac{\partial}{\partial \zeta} \\
& +\frac{\partial \zeta \circ T^{\varphi}}{\partial \bar{w}^{j}} \frac{\partial}{\partial \bar{\zeta}}, \quad(j=1, \cdots, n) .
\end{aligned}
$$

However from $h(z)=\exp \left(2 \pi \sqrt{-1} \sum_{j, 1=1}^{n} Q_{i j} z^{i} \bar{z}^{j}\right)$, it follows that

$$
\sum_{k=1}^{n} C_{\bar{j}}^{k} \frac{\partial \log h}{\partial z^{k}}=-2 \pi \sqrt{-1} \sum_{i, k=1}^{n} Q_{k \bar{j}} C_{\bar{i}}^{k} \bar{z}^{i}
$$

so that we find, for every $(w, t) \in C \times C$,

$$
\begin{aligned}
T_{*(u, t)}^{\varphi}\left(\frac{\partial}{\partial \bar{w}^{j}}\right)= & \left(\frac{\partial}{\partial \bar{z}^{j}}-\sum_{k=1}^{n} C_{\bar{j}}^{k} \frac{\partial}{\partial z^{k}}\right)_{T^{\varphi}(w, t)} \\
& +\left\{\left(\sum_{k=1}^{n} C_{\bar{j}}^{k} \frac{\partial \log h}{\partial z^{k}}+a_{j}\right) \zeta \frac{\partial}{\partial \zeta}\right\}_{T^{\varphi}(w, t)}+\left(\frac{\partial \bar{\zeta}}{\partial \bar{w}^{j}} \frac{\partial}{\partial \bar{\zeta}}\right)_{T^{\varphi}(w, t)}, \\
& (j=1, \cdots, n)
\end{aligned}
$$

On the other hand it is clear that

$$
T_{*}^{\varphi}\left(\frac{\partial}{\partial \bar{t}}\right)=\frac{\partial \bar{\zeta} \circ T^{\varphi}}{\partial \bar{t}} \frac{\partial}{\partial \bar{\zeta}} .
$$

Then the above equations show 


$$
T_{\varphi}^{\prime \prime}\left(C^{n} \times C\right)=\left\{\left\{T_{*}^{\varphi}\left(\frac{\partial}{\partial \bar{w}^{1}}\right), \cdots, T_{*}^{\varphi}\left(\frac{\partial}{\partial \bar{w}^{n}}\right), T_{*}^{\varphi}\left(\frac{\partial}{\partial \bar{t}}\right)\right\}\right\}
$$

\section{§5. The main theorem}

We shall complete in this section the proof of the following.

Theorem 5.1. Let $T$ be an abelian variety of complex dimension $n$ $(n \geqq 2)$, and let $B$ be a negative line bundle over $T$. We denote by $(B, T)$ the isolated singularity defined from $B$ and $T$, as stated at 2.2 in $\S 2$. Then any local deformation of $(B, T)$ is also $\left(B^{\prime}, T^{\prime}\right)$, where $T^{\prime}$ is an abelian variety of $\operatorname{dim}_{c} T^{\prime}=n$, and $B^{\prime}$ represents a negative line bundle over $T^{\prime}$.

First of all recall that the infinitesimal deformation $H^{1}\left({ }^{\circ} T^{\prime \prime}\left(B_{1}\right)\right)$ of $(B, T)$ is isomorphic to $\mathscr{H}^{1}$ as in the previous section, and that any element of $\mathscr{H}^{1}$ is integrable (c.f. Proposition 4.1.). Therefore we shall at first prove that small $C R$-structures in $\mathscr{H}^{1}$ are induced ones from negative line bundles over abelian varieties.

Now let us take any element $\varphi$ of $\mathscr{H}^{1}$ with $\varphi\left(Z_{\bar{j}}\right)=\sum_{k=1}^{n} C_{j}^{k} Z_{k}-$ $\sqrt{-1} a_{j}(\partial / \partial \theta),(j=1, \cdots, n)$, whose norm $|\varphi|$ is sufficiently small, and fix $\varphi$. Then the $C R$-structure ${ }^{\varphi} T^{\prime \prime}\left(C^{n} \times S^{1}\right)$ on $C^{n} \times S^{1}$ defined by (4.2) is induced from the complex structure $T_{\varphi}^{\prime \prime}\left(C^{n} \times C\right)$ on $C^{n} \times C$ determined in terms of (4.3) in Proposition 4.2. Moreover from Proposition 4.3, $T_{\varphi}^{\prime \prime}\left(C^{n} \times C\right)$ arises out of the standard complex structure on $C^{n} \times C$ by the map $T^{\varphi}: C^{n} \times C$ $\rightarrow C^{n} \times C$ which is defined by (4.5). We regard this map $T^{\varphi}$ as the bundle map between two trivial bundles $C^{n} \times C$ over $C^{n}$. Thus in order to prove the above statement it is enough to show that there exist an abelian variety $T^{\prime}$ and a negative line bundle $B^{\prime}$ over $T^{\prime}$ such that the bundle map $T^{\varphi}: C^{n} \times C \rightarrow C^{n} \times C$ induces canonically a bundle map $\tilde{T}^{\varphi}: B \times B$.

From now on we shall show the above statements. For this aim let us put

$$
C=\left(\begin{array}{c}
C_{1}^{1}, \cdots, C_{\bar{n}}^{1} \\
\ldots, \ldots, C_{\bar{n}}^{n}
\end{array}\right), \quad a=\left(\begin{array}{c}
a_{1} \\
\vdots \\
C_{\frac{1}{n}}^{n}, \cdots \\
a_{n}
\end{array}\right)
$$

and

$$
Q=\left(\begin{array}{c}
Q_{1 \overline{1}}, \cdots, Q_{1 \bar{n}} \\
\ldots \cdots \cdots \\
Q_{n \overline{1}}, \cdots, Q_{n \pi}
\end{array}\right)
$$


Then the condition (3.15) becomes

$$
{ }^{t} C Q={ }^{t} Q C
$$

where $t$ denotes the transpose of matrices. Furthermore the map $T^{\varphi}$ is also represented as follows;

$$
\begin{aligned}
& T^{\varphi}(w, t)=(w-C-\bar{w}, t \cdot \exp \{ 2 \pi \sqrt{-1} \bar{w}^{t} Q C \bar{C} w \\
&\left.\left.-\sqrt{-1} \pi^{t} \bar{w}^{t} Q C \bar{w}+{ }^{t} a \bar{w}\right\}\right),
\end{aligned}
$$

where

$$
w=\left(\begin{array}{c}
w^{1} \\
\vdots \\
w^{n}
\end{array}\right) \in C^{n}
$$

Here we set

$$
g(w)=\exp \left\{2 \pi \sqrt{-1} \bar{w}^{t} Q C \bar{C} w-\sqrt{-1} \pi^{t} \bar{w}^{t} Q C \bar{w}+{ }^{t} a \bar{w}\right\} .
$$

so that it follows that

$$
T^{\varphi}(w, t)=(w-C \bar{w}, g(w) t) .
$$

Now we write $\omega^{\prime}$ and $f^{\prime}(w, d)$ a periodic matrix and an automorphic factor respectively, corresponding to an abelian variety $T^{\prime}$ and a negative line bundle $B^{\prime}$ over $T^{\prime}$ to be required for the given $C R$-structure $\varphi \in \mathscr{H}^{1}$. Then since $T^{\varphi}$ in (5.2) induces a bundle map of $B^{\prime}$ onto $B, \omega^{\prime}$ and $f^{\prime}$ must satisfy the following conditions;

$$
\omega^{\prime}-c \bar{\omega}^{\prime}=\omega
$$

and

$$
\begin{aligned}
& g\left(w+\omega^{\prime} d\right) f^{\prime}(w, d)=g(w) f(w-C \bar{w}, d), \\
& \quad \text { for all }(w, d) \in C^{n} \times Z^{2 n},
\end{aligned}
$$

where $\omega$ is the periodic matrix of $T$ and $f$ denotes the automorphic factor of $B$ in $\S 2$. Since $|\varphi|$ is sufficiently small, there exists a unique $\omega^{\prime}$ satisfying (5.3), and we fix $\omega^{\prime}$. Furthermore we obtain the following.

Proposition 5.2. Let $f^{\prime}$ be the map of $C^{n} \times Z^{2 n}$ into $C$ defined by (5.4). Then $f^{\prime}$ becomes an automorphic factor for the periodic matrix, $\omega^{\prime}$, that is,

$$
f^{\prime} \text { is the holomorphic map of } C^{n} \times Z^{2 n} \text { into } C-\{0\} \text {, }
$$


and

$$
\begin{aligned}
& f^{\prime}\left(w, d_{1}+d_{2}\right)=f^{\prime}\left(w+\omega^{\prime} d_{1}, d_{2}\right) f^{\prime}\left(w, d_{1}\right), \\
& \text { for } w \in C^{n} \text { and } \boldsymbol{d}_{i} \in Z^{2 n}(i=1,2) .
\end{aligned}
$$

The proof of this proposition is due to the following two lemmas.

Lemma 5.3. For an arbitrary $(w, d) \in C^{n} \times Z^{2 n}$, we have

$$
f(w, d)=f(w, d) g\left(\omega^{\prime} d\right)^{-1} \exp \left(-2 \pi \sqrt{-1}^{t}\left(\bar{\omega}^{\prime} d\right)^{t} Q C \bar{C} w\right\} .
$$

Proof. At the beginning we get, using (5.1), (5.3) and ${ }^{t} Q C={ }^{t} C Q$,

$$
\begin{array}{r}
g\left(w+\omega^{\prime} d\right)=g(w) g\left(\omega^{\prime} d\right) \exp \left\{2 \pi \sqrt{-1} t \bar{w}^{t} Q C\left(\bar{C} \omega^{\prime}-\bar{\omega}^{\prime}\right) d\right. \\
\left.+2 \pi \sqrt{-1}\left(\bar{\omega}^{\prime} d\right)^{t} Q C \bar{C} w\right\} .
\end{array}
$$

Therefore it follows from (5.4) that

$$
\begin{aligned}
f^{\prime}(w, d)=f(w-C \bar{w}, d) g\left(\omega^{\prime} d\right)^{-1} \exp \left\{-2 \pi \sqrt{-1} \bar{w}^{t} Q C\left(\bar{C} \omega^{\prime}-\bar{\omega}^{\prime}\right) d\right. \\
\left.-2 \pi \sqrt{-1^{t}}\left(\bar{\omega}^{\prime} d\right)^{t} Q C \bar{C} s\right\} .
\end{aligned}
$$

On the other hand we find from (2.1)

$$
f(w-C \bar{w}, d)=\exp \left\{2 \pi \sqrt{-1}^{t}(w-C \bar{w}) Q \bar{\omega} d+A(d)\right\},
$$

where we put $A(d)=\frac{1}{2}^{t} \boldsymbol{d} A^{\circ} \boldsymbol{d}+{ }^{t} \boldsymbol{b} \boldsymbol{d}$, so that we obtain

$$
\begin{aligned}
f^{\prime}(w, d)= & g\left(\omega^{\prime} d\right)^{-1} \exp \left\{-2 \pi \sqrt{-1^{t}}\left(\bar{\omega}^{\prime} d\right)^{t} Q C \bar{C} w\right\} \\
& \times \exp \left\{-2 \pi \sqrt{-1} \bar{w}^{t} Q C\left(\bar{C} \omega^{\prime}-\bar{\omega}^{\prime}\right) d\right\} \\
& \times \exp \left\{2 \pi \sqrt{-1} t(w-C \bar{w}) Q\left(\bar{\omega}^{\prime}-\bar{C} \omega^{\prime}\right) d+A(d)\right\} \\
= & g\left(\omega^{\prime} d\right) \exp \left\{-2 \pi \sqrt{-1} t\left(\bar{\omega}^{\prime} d\right)^{t} Q C \bar{C} w\right\} f(w, d) .
\end{aligned}
$$

Moreover we have

LEMMA 5.4. $f^{\prime}$ satisfies (5.6).

Proof. From (5.7) it is clear that

$$
\begin{aligned}
f^{\prime}\left(w, d_{1}+d_{2}\right)= & f\left(w, d_{1}+d_{2}\right) g\left(\omega^{\prime}\left(d_{1}+d_{2}\right)\right)^{-1} \\
& \times \exp \left\{-2 \pi \sqrt{-1}^{t}\left(\bar{\omega}^{\prime}\left(\boldsymbol{d}_{1}+\boldsymbol{d}_{2}\right)\right)^{t} Q C \bar{C} w\right\} .
\end{aligned}
$$

Using the fact that $f$ is the automorphic factor, we get from (5.8)

$$
\begin{aligned}
g\left(\omega^{\prime} d_{1}+\omega^{\prime} d_{2}\right)= & g\left(\omega^{\prime} d_{1}\right) g\left(\omega^{\prime} d_{2}\right) \exp \left\{2 \pi \sqrt{-1} t\left(\bar{\omega}^{\prime} d_{1}\right)^{t} Q C\left(\bar{C} \omega^{\prime}-\bar{\omega}^{\prime}\right) d_{2}\right\} \\
& \left.+2 \pi \sqrt{-1}\left(\bar{\omega}^{\prime} d_{2}\right)^{t} Q C \bar{C} \omega^{\prime} d_{1}\right\} .
\end{aligned}
$$

Hence it follows that 


$$
\begin{aligned}
f^{\prime}(w, & \left.d_{1}+d_{2}\right)=f\left(w+\omega d_{1}, d_{2}\right) f\left(w, d_{1}\right) g\left(\omega^{\prime} d_{1}\right)^{-1} g\left(\omega^{\prime} d_{2}\right)^{-1} \\
& \times \exp \left(-2 \pi \sqrt{-1^{t}}\left(\bar{\omega}^{\prime} d_{1}\right)^{t} Q C\left(\bar{C} \omega^{\prime}-\bar{\omega}^{\prime}\right) d_{2}-2 \pi \sqrt{-1^{t}}\left(\bar{\omega} d_{1}\right)^{t} Q C \bar{C} \omega\right\} \\
& \times \exp \left\{-2 \pi \sqrt{-1^{t}}\left(\bar{\omega}^{\prime} d_{2}\right)^{t} Q C \bar{C}\left(w+\omega^{\prime} d_{1}\right)\right\} .
\end{aligned}
$$

But we get

$$
f\left(w+\omega^{\prime} d_{1}, d_{2}\right)=f\left(w+\omega^{\prime} d_{1}, d_{2}\right) \exp \left\{2 \pi \sqrt{-1^{t}}\left(-C \bar{\omega}^{\prime} d_{1}\right) Q\left(\bar{\omega}^{\prime}-\bar{C} \omega^{\prime}\right) d_{2}\right\} .
$$

Finally noting ${ }^{t}\left(C \bar{\omega}^{\prime} d_{1}\right) Q={ }^{t}\left(\bar{\omega}^{\prime} d_{1}\right)^{t} Q C$, we find in terms of (5.9) and the above relation,

$$
\begin{aligned}
f^{\prime}\left(w, d_{1}+d_{2}\right)= & f\left(w+\omega^{\prime} d_{1}, d_{2}\right) g\left(\omega^{\prime} d_{2}\right)^{-1} \\
& \times \exp \left\{-2 \pi \sqrt{-1}{ }^{t}\left(\bar{\omega}^{\prime} d_{2}\right)^{t} Q C \bar{C}\left(w+\omega^{\prime} \boldsymbol{d}_{1}\right)\right\} \\
& \times f\left(w, \boldsymbol{d}_{1}\right) g\left(\omega^{\prime} \boldsymbol{d}_{1}\right)^{-1} \exp \left\{-2 \pi \sqrt{-1^{t}}\left(\bar{\omega}^{\prime} \boldsymbol{d}_{1}\right)^{t} Q C \bar{C} w\right\} .
\end{aligned}
$$

This equation shows (5.6).

Q.E.D.

From the above lemmas, Proposition 5.2 is proved. Further let $T^{\prime}$ and $B^{\prime}$ the abelian variety and the line bundle over $T^{\prime}$, respectively, defined from $\omega^{\prime}$ and $f^{\prime}$ in Proposition 5.2. Then we have that following.

Proposition 5.5. Let all notations be as above. If $C$ is sufficiently small, then $B^{\prime}$ is negative. Moreover the map $T^{\varphi}: C^{n} \times C \rightarrow C^{n} \times C$ defined by (4.5) (or (4.5)') induces canonically the bundle map $\tilde{T}^{\varphi}: B^{\prime} \rightarrow B$.

Proof. This is trivial from constructions of $T^{\varphi}$ and $B^{\prime}$.

Q.E.D.

Thus all small $C R$-structures in $\mathscr{H}^{1}$ are induced ones from negative line bundles over abelian varieties.

Now from the universality theorem in $\S 9$ [1], we know the following facts; Let $\iota_{B_{1}}: B_{1} \rightarrow B$ be the inclusion map as before and let $N$ be any neighborhood of $B_{1}$ in $B$. When $N_{\omega}$ is a deformation of the complex structure on $N$ which is the open submanifold of $B$, where $\omega \in \Gamma\left(N, T^{\prime \prime}(N)^{*}\right.$ $\left.\otimes T^{\prime}(N)\right)$ and an embedding $i: B_{1} \rightarrow N$ is given, we denote by $\omega \circ i$ the induced $C R$-structure on $V$ by $i: B_{1} \rightarrow N$. Finally let $\mathscr{H}_{K}^{1}$ be the harmonic space in $\Gamma\left({ }^{\circ} T^{\prime \prime}\left(B_{1}\right) * \otimes T^{\prime}\left(B_{1}\right)\right)$ with respect to the operator $\bar{\partial}_{b}^{(2) *} \bar{\partial}_{b}^{(1)}+\bar{\partial}_{b}^{(0)} \bar{\partial}_{b}^{(1) *}$ $\left(\operatorname{dim}_{R} \mathscr{H}_{R}^{1}<\infty\right)$. Then there exists a differential map

$\psi_{K}: \mathscr{H}_{K}^{1} \rightarrow \Gamma\left({ }^{\circ} T^{\prime \prime}\left(B_{1}\right) * \otimes T^{\prime}\left(B_{1}\right)\right)$ satisfying the following conditions;

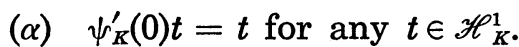

(B) If $N_{\omega}$ is a deformation of $N$ such that $n_{1}$-Sobolev-norm $\|\omega\|_{n_{1}}$ of $\omega$ is sufficiently small ( $n_{1}$ is a sufficiently large integer), then there are a point $t_{\omega} \in \mathscr{H}_{K}^{1}$ and an embedding $i_{\omega}: B_{1} \rightarrow N_{\omega}$ such that 


$$
\omega \circ i_{\omega}=\psi_{K}\left(i_{\omega}\right) \text {. }
$$

Moreover $i_{\omega}$ and $t_{\omega}$ are infinitely differentiable on $\omega$, and when $\omega$ is zero, it follows that $t_{\omega}=0$ and $\omega \circ{ }^{i} \omega=\iota_{V}$.

Here we can prove the following

Proposition 5.6. For any sufficiently small point $t \in \mathscr{H}_{K}^{1}, \psi_{K}(t)$ is an induced $C R$-structure on $V$ form a negative line bundle over an abelian variety.

Proof. At first, for any small $\varphi \in \mathscr{H}^{1}$ with the expression (4.1) we have shown that the complex structure $T_{\varphi}^{\prime \prime}$ on $B$ defined by (4.3) becomes one of a negative line bundle $B^{\prime}$ over an abelian variety $T^{\prime}$ (c.f. Proposition 5.5) and that the $C R$-structure is induced from $B^{\prime}$. Here let $\hat{T}$ be the smooth map of $\mathscr{H}^{1}$ into $\Gamma\left(T^{\prime \prime}(B)^{*} \otimes T^{\prime}(B)\right)$ defined by

$$
\begin{aligned}
& \hat{T}(\varphi)\left(\frac{\partial}{\partial \bar{z}^{j}}\right)=\sum_{k=1}^{n} C_{j}^{k} \frac{\partial}{\partial z^{k}}+\left(\sum_{k=1}^{n} \frac{\partial \log h}{\partial z^{k}}-a_{j}\right) \zeta \frac{\zeta}{\partial \zeta}, \quad(j=1, \cdots, n) \\
& \hat{T}(\varphi)\left(\frac{\partial}{\partial \bar{\zeta}}\right)=0
\end{aligned}
$$

for any $\varphi \in \mathscr{H}^{1}$ with (4.1), (see (4.3)). Moreover let $\mathscr{H}^{1}$ be a sufficiently small neighborhood of 0 in $\mathscr{H}^{1}$ such that Proposition 5.5 and the above statement $(\beta)$ hold good. Then we have from $(\beta)$ a smooth map

$$
\tau: \mathscr{H}_{e}^{1} \longrightarrow \mathscr{H}_{K}^{1} \quad \text { such that } \hat{T}(\varphi) \circ i_{T(\varphi)}=\psi_{K}(\tau(\varphi)) \text { and } \tau(0)=0 .
$$

In order to prove this proposition it is enough to show that the derivation $\tau_{(0)}^{1}$ of $\tau$ at 0 is injective, because of $\operatorname{dim} \mathscr{H}^{1}=\operatorname{dim} \mathscr{H}_{K}^{1}$. Here let $s$ be a small real number. Then it follows from Theorem 7.1 and (5.16) in [1] that

$$
\lim _{s \rightarrow 0} \frac{\hat{T}(s \varphi) \circ i_{T(s \varphi)}-\hat{T}(s \varphi) \circ \iota B_{1}}{s}=\bar{\partial}_{b}^{(0)} \xi
$$

where $\xi$ is an element of $\Gamma\left(T^{\prime}\left(B_{1}\right)\right)$.

On the other hand we get from the definition of $\hat{T}$

$$
\lim _{s \rightarrow 0} \frac{\hat{T}(s \varphi) \circ B_{1}-\hat{T}(0) \circ \iota B_{1}}{s}=\varphi .
$$

Therefore we have from (5.10) and (5.11)

$$
\left.\frac{d \hat{T}(s \varphi) \circ i_{T(s \varphi)}}{d s}\right|_{s=0}=\varphi+\bar{\partial}_{b}^{(0)} \xi \text {. }
$$


Furthermore it follows, using $(\beta)$, that

$$
\left.\frac{d \psi_{K}(\tau(s \varphi))}{d s}\right|_{s=0}=\tau^{\prime}(0) \cdot \varphi
$$

so that

$$
\tau^{\prime}(0) \cdot \varphi=\bar{\partial}_{b}^{(0)} \xi+\varphi .
$$

Now suppose that $\tau^{\prime}(0) \cdot \varphi=0$. Then it is clear from (5.12) that the $\bar{\partial}_{b}^{(1)}$-cohomology class of $\varphi$ is zero, but as $\varphi$ belongs to $\mathscr{H}^{1}$ we have $\varphi=0$. Thus $\tau^{\prime}(0)$ is injective.

Q.E.D.

Our main Theorem 5.1 is obtained from the above Proposition 5.6.

\section{REFERENCES}

[1] M. Kuranishi, Deformations of isolated singularities and $\bar{\partial}_{b}$, Preprint, Columbia Univ. (1973).

[ 2 ] M. Schlessinger, On rigid singularities, Rice Univ. Studies 59 (1973), 147-162.

[3] G. N. Tjurina, Locally semiuniversal flat deformations of isolated singularities of complex spaces, Izv. Akad. Nauk SSSR Ser. Mat. 33 (1970), 1026-1058.

[4] T. Akahori, Intrinsic formula for Kuranishi's, to appear in Publications Res. Inst. Math. Sciences, Kyoto Univ.

[5] A. Weil, Variétés Kahlériennes, Hermann, Paris, (1958).

[6] H. Grauert, Über Modifikationen und exzeptionelle analytische Mengen, Math. Ann. 146 (1962), 331-368.

Nagoya University

and

Kyoto University 